Portland State University

PDXScholar

Spring 6-15-2018

\title{
Biomimetic Design and Construction of a Bipedal Walking Robot
}

Alexander Gabriel Steele

Portland State University

Follow this and additional works at: https://pdxscholar.library.pdx.edu/open_access_etds

Part of the Biomechanics Commons, Mechanical Engineering Commons, and the Robotics Commons Let us know how access to this document benefits you.

\section{Recommended Citation}

Steele, Alexander Gabriel, "Biomimetic Design and Construction of a Bipedal Walking Robot" (2018). Dissertations and Theses. Paper 4486.

https://doi.org/10.15760/etd.6370

This Thesis is brought to you for free and open access. It has been accepted for inclusion in Dissertations and Theses by an authorized administrator of PDXScholar. Please contact us if we can make this document more accessible: pdxscholar@pdx.edu. 
Biomimetic Design and Construction of a Bipedal Walking Robot

by

Alexander Gabriel Steele

A thesis submitted in partial fulfillment of the requirements for the degree of

Master of Science

in

Mechanical Engineering

Thesis Committee:

Alexander Hunt, Chair

David Turcic

Derek Tretheway

Portland State University

2018 
(C) 2018 Alexander Gabriel Steele 


\begin{abstract}
Human balance and locomotion control is highly complex and not well understood. To understand how the nervous system controls balance and locomotion works, we test how the body responds to controlled perturbations, the results are analyzed, and control models are developed. However, to recreate this system of control there is a need for a robot with human-like kinematics. Unfortunately, such a robotic testbed does not exist despite the numerous applications such a design would have in mobile robotics, healthcare, and prosthetics.

This thesis presents a robotic testbed model of human lower legs. By using MRI and CT scans, I designed joints that require lower force for actuation, are more wear resistant, and are less prone to catastrophic failure than a traditional revolute (or pinned) joints. The result of using this process is the design, construction, and performance analysis of a biologically inspired knee joint for use in bipedal robotics.
\end{abstract}

For the knee joint, the design copies the condylar surfaces of the distal end of the femur and utilizes the same crossed four-bar linkage design the human knee uses. The joint includes a changing center of rotation, a screw-home mechanism, and patella; these are characteristics of the knee that are desirable to copy for bipedal robotics. The design was calculated to have an average sliding to rolling ratio of 0.079 , a maximum moment arm of 2.7 inches and a range of motion of 151 degrees. This should reduce joint wear and have kinematics similar to the human knee. I also designed and constructed novel, adjustably-damped hip and ankle joints that use braided pneumatic actuators. These joints 
provide a wide range of motion and exhibit the same change in stiffness that human joints exhibit as flexion increases, increasing stability, adaptability, and controllability.

The theoretical behaviors of the joints make them desirable for use in mobile robotics and should provide a lightweight yet mechanically strong connection that is resistant to unexpected perturbations and catastrophic failure. The joints also bridge the gap between completely soft robotics and completely rigid robotics. These joints will give researchers the ability to test different control schemes and will help to determine how human balance is achieved. They will also lead to robots that are lighter and have lower power requirements while increasing the adaptability of the robot. When applying these design principles to joints used for prosthetics, we reduce the discomfort of the wearer and reduce the effort needed to move. Both of which are serious issues for individuals who need to wear a prosthetic device. 


\section{Dedication}

To my Uncle, if I ever became half the man you were I would be proud. Also, to my cats, without whom I would have been able to finish this work in half the time and with far less cat hair everywhere. 


\section{Acknowledgements}

The author would like to extend a heartfelt thanks to Derek Tretheway. Whom, without his understanding, none of this would have been possible. I cannot even imagine where I would be if he had not taken a chance on me; I will never forget it.

Thanks to Alexander Hunt, for giving me the freedom to create the things I imagine, for giving me a chance to work with and learn from him, and for the opportunity to build something amazing, despite my somewhat embarrassing and awkward first introduction.

Finally, to the rest of the lab and specifically Wade Hilts, may the horses of Nazareth always ride in your favor. 


\section{Contents}

Abstract

Dedication iii

Acknowledgements $\quad$ iv

List of Tables $\quad$ vii

List of Figures $\quad$ viii

$\begin{array}{ll}\text { Chapter } 1 \text { Introduction } & 1\end{array}$

1.1 - Current robotic joint types and limitations 2

$\begin{array}{ll}\text { Chapter } 2 \text { Objectives } & 7\end{array}$

$\begin{array}{lr}\text { Chapter } 3 \text { Background } & 8\end{array}$

3.1 - Anatomy of Synovial Joints $\quad 8$

3.2 - Hip joint anatomy and physiology 10

3.2 - Knee joint anatomy and physiology 15

3.3 - Ankle and foot anatomy and physiology 18

3.3.1 - Ankle anatomy and physiology 19

3.3.2 - Foot anatomy and physiology 20

3.4 - 3D printing with continuous fiber $\quad 21$

$\begin{array}{ll}\text { Chapter } 4 \text { Methods and Materials } & 23\end{array}$

4.1 - Medical scans to 3D models 23

4.2 - Obtaining medical scans 24

4.3 - Creating a 3D model $\quad 25$ 
4.4 - Hip Joint 30

4.5 - Knee Joint $\quad 34$

4.5.1 - Version 1

4.5.2 - Version 2

4.5.3 - Version $3 \quad 40$

4.5.4 - Version $4 \quad 42$

4.6 - Ankle Joint and foot 45

4.6.1 - Version $1 \quad 45$

4.6.2 - Version $2 \quad 47$

Chapter 5 Results 49

5.1 - Hip Joint Results 49

5.2 - Knee Joint Results 53

5.2.1 - Version 1

5.2.2 - Version $2 \quad 55$

5.2.3 - Version $3 \quad 62$

$\begin{array}{ll}5.3 \text { - Ankle and foot } & 68\end{array}$

$\begin{array}{ll}5.3 .1-\text { Version } 1 & 68\end{array}$

$\begin{array}{ll}5.3 .2 \text { - Version } 2 & 70\end{array}$

$\begin{array}{ll}\text { Chapter } 6 \text { Discussion } & 73\end{array}$

$\begin{array}{ll}\text { Bibliography } & 77\end{array}$ 


\section{List of Tables}

Table 1 - Range of motion upper and lower bounds for the human hip joint [16].......... 14

Table 2 - Material properties for continuous fiber inlay used in the MarkForged Mark II

3D printer provided from the company. *Measured by a method similar to ASTM D790

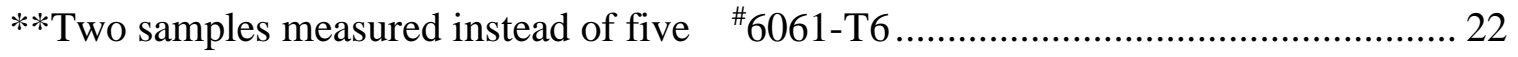

Table 3 - Range of motion of the hip joint for the average adult male ........................... 53

Table 4 - Average ankle joint range of motion ....................................................... 75 


\section{List of Figures}

Figure 1 - Design of a rolling knee (RK) joint............................................................. 4

Figure 2 - Cutaway of a ball and socket synovial joint ............................................ 9

Figure 3 - The three degrees of freedom of the hip joint ........................................ 10

Figure 4 - Anatomy of the hip joint as viewed anteriorly. The femoral head (ball) fits into the acetabulum (socket), which is held in place by the joint capsule. ........................... 11 Figure 5 - Lateral view of the hip joint showing the ligamentum teres (cut into two

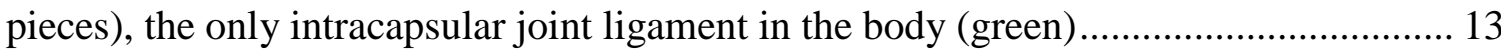

Figure 6 - Measuring points used to determine the quadriceps angle (q-angle).............. 14

Figure 7 - The six degrees of freedom that make up the human knee ............................ 16

Figure 8 - The 4 main ligaments of the human knee (not included the PFL and ALL) ... 16 Figure 9 - The tibia and fibula form a square shaped bracket that fits around the heart-

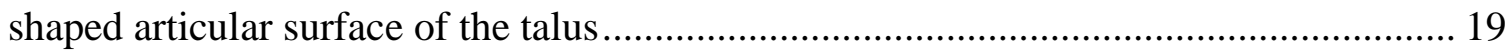
Figure 10 - Cutaway of a 3D printed part showing the continuous Kevlar fiber inlay (yellow) inside the nylon and chopped carbon fiber composite (black) ........................ 21

Figure 11 - DICOM library search tool layout via 3D Slicer ...................................... 25

Figure 12 - DICOM files loaded into 3D slicer as viewed in the GUI ......................... 27

Figure 13 - Results of a CT scan (left) vs. an MRI scan (right) notice that the CT scan shows a much higher contrast for bones than the MRI scan...................................... 28

Figure 14 - Initial attempt as selecting bone exclusively using the software's selection

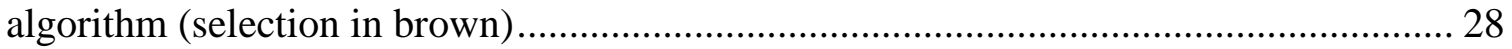


Figure 15 - Bone selection (brown) after manual cleanup and verification of selection .. 29 Figure 16 - Initial result from the extrude operation using 3D Slicer. This operation creates a "stepped" effect that needs to be later smoothed.

Figure 17 - (A) The 3D model from 3D Slicer loaded into Mesh Mixer and (B) after the

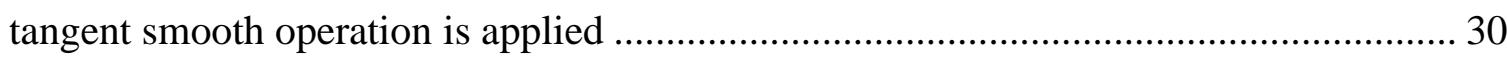

Figure 18 - Hip Model created using the extrude function in 3D Slicer........................ 31

Figure 19 - Geometric considerations for designing the hip joint and femur .................. 32

Figure 20 - 3D model of the designed femur....................................................... 33

Figure 21 - Anterior view of the 3D model of the designed hip with vertebrae.............. 34

Figure 22 - 3D model of the first version of the knee joint ..................................... 36

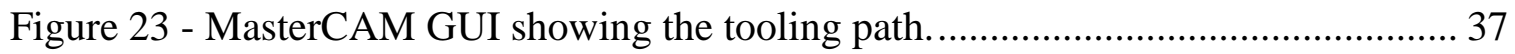

Figure 24 - Machining of the tibial head using a 3-axis CNC machine ......................... 37

Figure 25 - Aluminum slug being machined for the tibial end using a 2-axis CNC

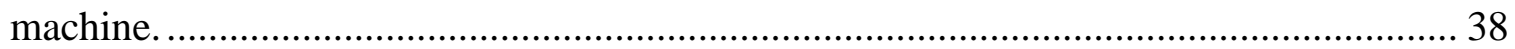

Figure 26 - Initial completion of the knee joint prior to welding …........................... 38

Figure 27 - Modification of the femoral end to reduce weight.................................... 39

Figure 28 - Tibial head showing the addition of the screw-home mechanism ............... 40

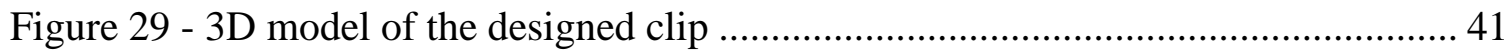

Figure 30 - 3D model showing the addition of locking surfaces on the fibular end........ 41

Figure 31 - The modification of the tibial head to allow for upright locking ................. 43

Figure 32 - Force applied to the knee through the patella as seen laterally ..................... 45

Figure 33 - 3D model of the first version of the foot and ankle ................................ 46 
Figure $34-3 \mathrm{D}$ rendering of the underside of the designed foot showing the addition of a lever arm to apply force to the constructed foot the same way the calcaneus does in the human foot (circled)

Figure $35-3 \mathrm{D}$ model of version 2 of the foot as seen laterally (and without the pneumatic sleeve, that attaches the joints)

Figure 36 - By combining the talus and calcaneus, the pneumatic spring element used for the arch is now protected. 48

Figure 37 - Lateral view of the pelvis showing several vertebrae and better illustrating the connecting surfaces between the sacrum and ilium 49 Figure 38 - Parameters for determining the single thread length of the braided sleeve and the number of turns for that thread. 51 Figure 39 - Calculated change in stiffness as it relates to joint angle at an initial pressure of 25 psi. 52 Figure 40 - Version one of the knee prototype showing how the pneumatic actuator changes as the knee rotates Figure 39 shows the assembled joint in different states of flexion 54

Figure 41 - Fully assembled second version of the knee joint with the cylinder inflated. 55 Figure 42 - 3D printed femoral end with the clips removed from the end 56 Figure 43 - 3D printed femoral end with the clips inlayed with Kevlar added back into the femoral end 56 Figure 44 - Second version of the 3D printed knee showing locking mechanism (circled) 
Figure 45 - Theoretical torque requirement to move a 46-kilogram load over the angle of rotation (blue solid line) vs the torque required for a pin joint (red dashed line) .............58

Figure 46 - Kinematics of sliding to rolling ratio from initial contact point $\mathrm{C} 1$ to $\mathrm{C} 2 \ldots . .59$

Figure 47 - Sliding and rolling ratio of the proposed joint. Note, the initially high sliding

ratio settles because the model settles prior to the start of the rotation.

Figure 48 - Radius arm $\mathrm{r}(\theta)$ changing as a function of the angle of rotation $\theta$ plotted against the radius arm of a pin joint (red-hashed line)

Figure 49 - Tibial head changes as the prototype testing progressed

Figure 50 - Tibial head seen laterally (A) prior to modification, (B) post modification, change highlighted in red circle, and (C) tibial head locked with the distal end of the femur for reference. 63 Figure 51 - Constructed joint (A) rear view, (B) front view, (C) full flexion, and (D) full extension 63 Figure 52 - Four-bar linkage used in the proposed design showing link designation, angle designation, and the coordinate system for the following equations. 64 Figure 53 - Instantaneous center of rotation plot of proposed joint for the full range of motion. 66

Figure 54 - Kinematics of sliding to rolling ratio from initial contact point C1 to C2 .... 67 Figure 55 - Sliding to rolling ratio over the designed joints range of motion where zero is a pure rolling condition and one is a pure sliding condition, which increases wear......... 68 Figure 56 - 3D printed version 1 of the foot as seen laterally 69 
Figure 57 - Distal view of version 1 of the 3D printed foot showing the lever arm addition



Figure 58 - Anterior view of the 3D printed version 1 foot showing the two toes .......... 70

Figure 59 - Foot design version 2 seen laterally after the assembly using the pneumatic

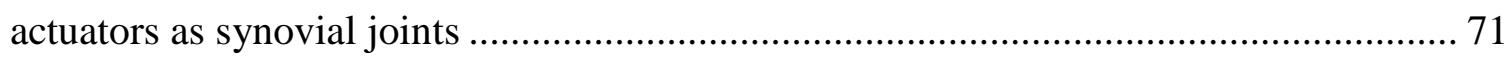

Figure 60 - Section view of the talus in SolidWorks showing the tube inside the model

that lets air flow down into the other joints of the foot........................................... 71

Figure 61 - 3D Printed foot version 2 with the pneumatic actuator sleeve for the ankle

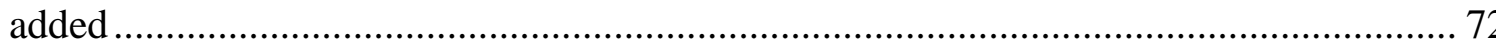




\section{Chapter 1}

Introduction

When the public thinks about robotics, in particular bipedal humanoid robots, science fiction movies have taken the forefront. Given the state of technology, it may even seem that such advanced robots are only years away, if not, already here. However, in 2015 the DARPA Robotics Challenge destroyed those dreams by highlighting, at the time, the best and most well-funded robotic teams from around the world.

These (mostly) bipedal robots, on average, took almost an hour to do what humans could accomplish in just a few minutes, if the robots could accomplish it at all. Ironically, these robots performed better than DARPA expected. This public failure to match expectations set by the movie industry brought to light the reality of robotics in the current state.

The reality is that robots are not agile, cannot easily adapt to the environment they are placed in, and if they fell over, could not recover. The spurious idea of robots performing, as well as, or better than humans was now replaced with the reality; even the best robots were close to helpless.

Robots that easily adapt to their surroundings have numerous applications; in search and rescue, these robots would be able to save lives of people without risking more lives in the process. Furthermore, these robots are able to go into environments that 
humans cannot, such as the Fukushima nuclear disaster, humanoid robots would be able to interact with the environment without exposing humans to the fatal radiation.

Robots need to be able to operate in an environment that was designed for human use. However, current mobile robots are designed like industrial machines and while this works for situations where extreme precision is needed, it does not allow them to adapt to changing environments in a way that would make them useful. For this reason, new designs for robotic joints that are more functional in a mobile platform are needed.

\section{1 - Current robotic joint types and limitations}

Currently, in robotics the most common type of joints used are revolute joints, also known as hinge joints or pin joints. There are several different examples of these joints, but they all have similar properties. This class of joints has a single degree of freedom, rotation about the pin. They are typically pinned center of the two joining rigid members. The single degree of freedom offered by pin joints makes them desirable for control purposes. Additionally, because of the simplicity of the joint, it is a low cost and easy to replace option.

However, due to the high shear stress that is applied to the joint through nor-mal operations, the joints wear quickly [1]. Clamping forces applied to the pin, or preload, also causes joint fatigue, which leads to premature wearing as it introduces tensile forces, and promotes creep. The ratio between the diameter of the hole and the edge of the material to the edge of the hole has a direct effect on the wear and failure of the joint 
because the stress concentration or bearing force is higher at the hole. Furthermore, failure of the joint is typically catastrophic and causes serious damage because the joint is a single pin without added supports.

In recent years, biomimetic design has led to several different attempts to model and design a mechanical joint similar to the human knee. These designs have looked at different aspects of the knee and attempted to mimic them. Several of these designs include screw-home and patellar analogs [2-5].

These designs often utilize the same simplified 4-bar linkage design created when modeling the human knee. However, the designs that have been put forth are "floating designs," or complex linkage designs that do not have contacting surfaces like those that the human knee utilizes. This predominately places load onto the pin connections and while the force profile over the entire range of motion differs, the pin joints have to carry the weight just as the solitary pin joint design first mentioned.

Lastly, Hobon et al. [6] modeled the benefits of using what they termed as a rolling knee joint (RK). This joint, (Fig. 1) consists of two rolling cylinders joined by a solid link, which has a pin joint at the center of either cylinder. This design eliminates a portion of the force placed on the pin joint by applying it through the contact point of the cylinders. The RK joint design reduces energy consumption during a walking gait when compared to a standard pin joint. 


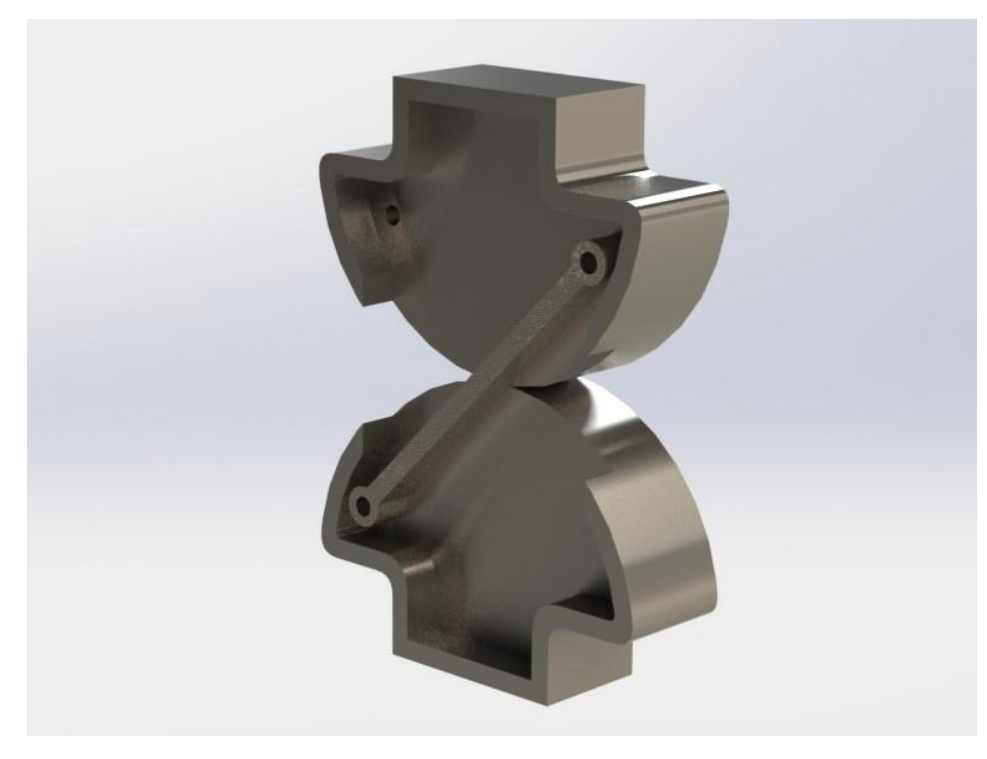

Figure 1 - Design of a rolling knee $(R K)$ joint

In the RK joint, the force from the weight of the robot is applied at a onedimensional line across the cylinder, this force leads to premature wearing, and furthermore an impact load of sufficient force causes permanent deformation of the cylinders.

Energy is needed to keep the robot upright when standing because the design lacks an upright locking mechanism. When fully flexed, the weight of the robot is still applied to the pin joints used to attach the two cylinders.

In this work, I propose a set of joints that will act as a lightweight, robust, and compact replacement to pin joints for the hip, knee, and ankle joints. These proposed designs draw on inspiration from the hip, knee, and ankle joints of humans; they were designed using MRI scans of the equivalent human joint. 
The joints use biological principles to imbue them with particular qualities that will offer them superior performance when compared to the standard robotic joint. Some of these qualities include locking in the upright position, much like the human knee as well as a changing moment arm that reduces the amount of force needed to create the same amount of torque using a pin joint.

In the next chapter, I will cover the objectives that I set out to accomplish in this thesis. These goals are what drove my research and properly explains the gap in knowledge that this thesis is filling.

Chapter 3 covers the anatomy and physiology of the joints in the lower half of the body. This chapter is an abridgment of what might be covered in biology, however understanding the main functions of the joints and how they are formed is needed for the subsequent chapters.

The fourth chapter discusses how to convert medical scans into 3D models, where to find medical scans, the capabilities of the MarkForged 3D printer that was used, as well as the design of the engineered joints. Each joint and, if applicable, the subordinate revisions to that joint are listed in order from oldest (version 1) to the newest.

Chapter 5 details the construction and theoretically predicted properties of the joints that were created which will be used later for testing, verification, and if needed, revision. As with Chapter 4, each major section covers a different joint, while each minor section covers the revisions to that joint. 
The final chapter discusses the results and limitations of this work. It also recommends future work that could be taken at a later time to improve upon the foundation that this thesis creates. 


\section{Chapter 2}

\section{Objectives}

The primary objective of this research is to develop a way to determine the in vivo kinematics of human joints from MRI and CT scans. Limited in vivo joint kinematic data exists because of the difficulty accurately tracking joint movement using external sensors. This data is important in the design of human inspired robotic joints because the human body is incredibly energy efficient and adaptable, however current robot designs are not.

The data will then be applied to the design of joints for a bipedal walking robot. Simulations will be done to determine both how energy efficient the designed joints are and how effective this new method is in determining human joint kinematics. 3D printed joints will then be constructed to verify the theoretical behavior and predicted kinematics.

This work will create a new methodology for designing mobile bipedal robotic joints that outperform the current designs.

This thesis breaks the designs into three chapter subsections, the hip joint, the knee joint, and the ankle joint. For the purposes of this thesis, the pelvis will be covered in the hip joint section and the foot will be included in the chapter covering the ankle joint and not separately. Each of these chapters covers the different versions that were proposed, designed, and/or tested. These chapters also discuss the advantages of each version, and if applicable, why the version was later modified in favor of its final form. 


\section{Chapter 3}

\section{Background}

This chapter covers the gross anatomy and physiology of the human joints that were used to model the designed robotic joints in this thesis. Understanding the basic biology of each joint will help clarify design decisions made in later chapters. As such, information about the biological structure of the joint, range of motion of the joint, and degrees of freedom of the joint is found here. Included in this chapter is information about the specialized 3D printer used to develop and test the mechanical joints, this information includes mechanical properties of the materials used and material testing results provided by the company.

\section{1 - Anatomy of Synovial Joints}

Synovial joints are the most common and most flexible type of joint in the human body [7]. Synovial joints join bones with a dense and fibrous capsule that is filled with synovial fluid or synovia. Synovia is a non-Newtonian fluid, which has the consistency of the white of an egg. This fluid acts as lubrication between the cartilage of the joint during movement [8].

There are six different classifications of synovial joints, however, the focus of this work is on the ball and socket type, such as the hip or shoulder and the hinge type, such as the ankle or the carpals of the wrist. Both of these joint types have distinct movement 
characteristics that are primarily defined by the articulating surfaces of the bones. The ball and socket type joint allows for three axes of rotation (flexion/ extension, abduction/adduction, and lateral/medial rotation), while hinge type joints only allow for flexion and extension in one plane [8-10].

All synovial joints are made up of a synovial cavity, a joint capsule, and articular cartilage (Fig. 2). The synovial cavity is the space between the bones that is filled with synovia.

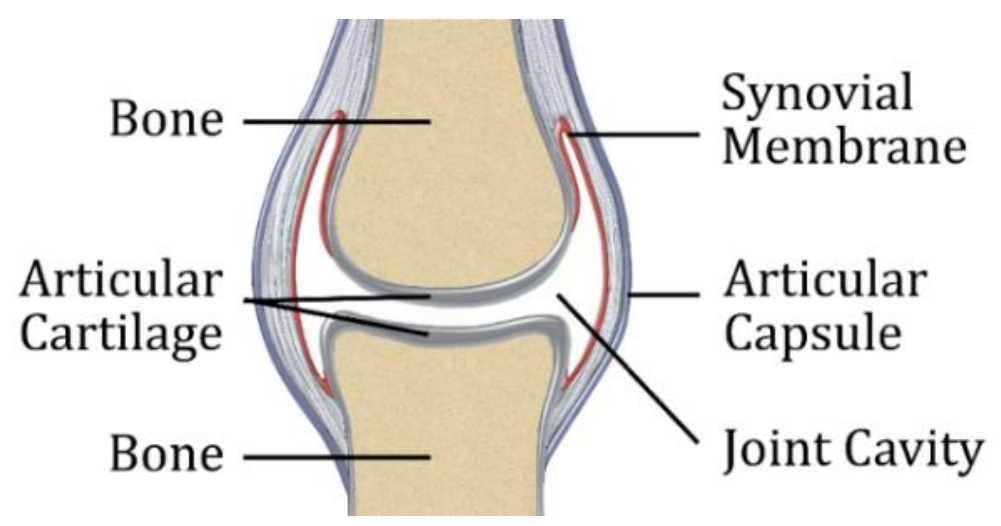

Figure 2 - Cutaway of a ball and socket synovial joint

The joint capsule is then further broken up into the outer layer and inner layer, the inner layer is made up of the synovial membrane, which secretes synovia, while the outer layer is a fibrous membrane that sometimes contains ligaments. The articular cartilage functions to reduce friction during movement and protects the joint from impact forces $[8,11,12]$. 


\section{2 - Hip joint anatomy and physiology}

The hip joint has three degrees of freedom, all rotational, (Fig. 3). The hip has no translational degrees of freedom.

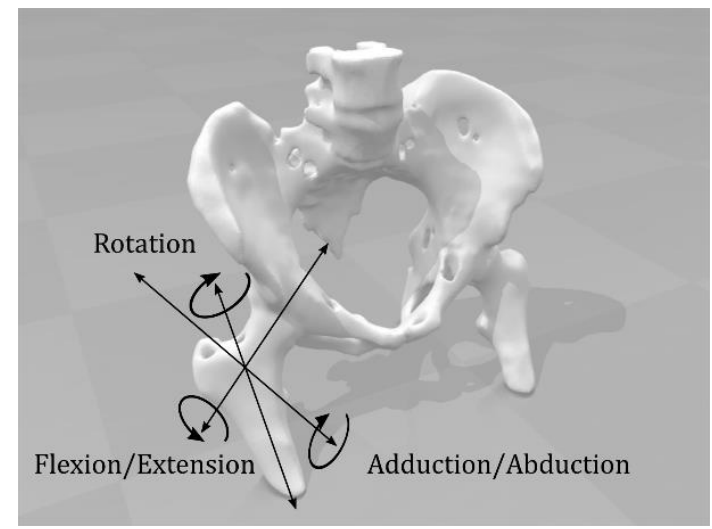

Figure 3 - The three degrees of freedom of the hip joint

The hip joint (Fig. 4) is a ball-and-socket synovial joint where the femoral head is the ball and the acetabulum creates a cup-like depression in which the femoral head fits [13]. 


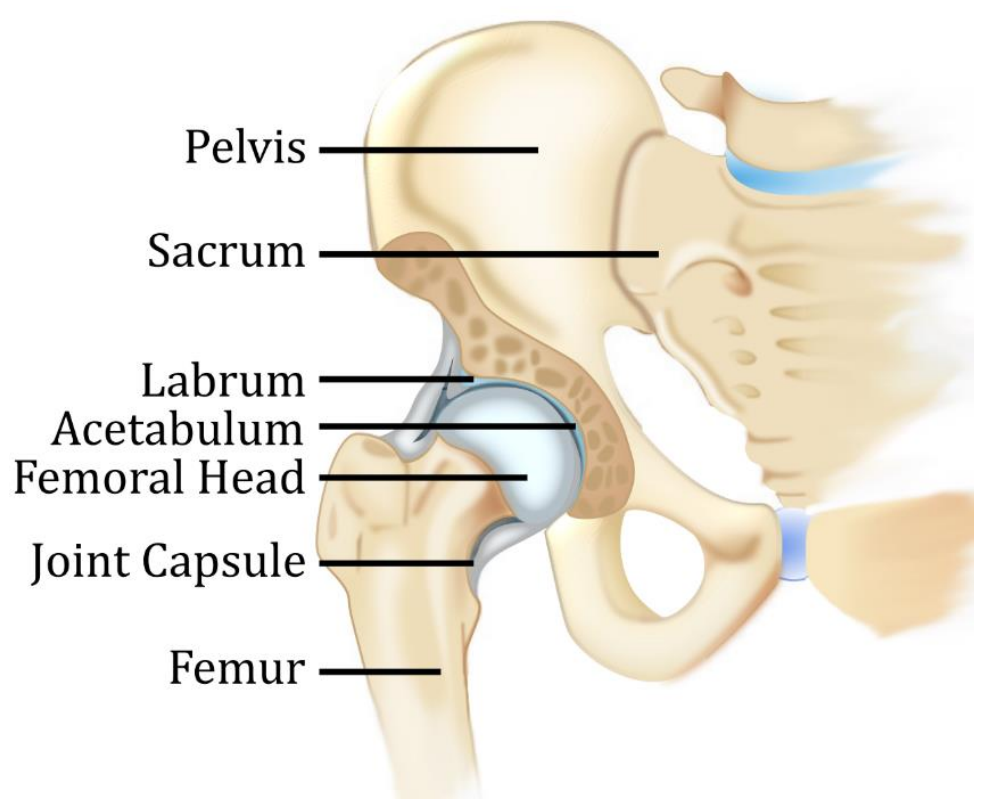

Figure 4 - Anatomy of the hip joint as viewed anteriorly. The femoral head (ball) fits into the acetabulum (socket), which is held in place by the joint capsule.

A synovial joint, as previously discussed, is characterized as a joint that is surrounded by a joint capsule. A joint capsule is a dense and fibrous structure that is analogous to tendons, but forms a sleeve around the joint. For the hip, the joint capsule is primarily formed from three ligaments:

1. Iliofemoral ligament

2. Pubofemoral ligament

3. ischiofemoral ligament

Ligaments are fibrous structures that connect bone-to-bone, similar to tendons, which are fibrous structures that connect muscle to bone. The iliofemoral ligament spans the distance between the anterior inferior iliac spine and the intertrochanteric line of the femur. It is "Y" shaped. This ligament prevents the hyperextension of the hip joint [13]. The pubofemoral ligament has a triangular shape and spans between the superior pubic 
rami and the intertrochanteric line of the femur. This ligament prevents excessive extension and abduction. Lastly, the ischiofemoral ligament spans between the body of the ischium and the greater trochanter of the femur. It has a spiral orientation and prevents excessive extension of the joint. These ligaments completely enclose the hip joint and form the joint capsule.

The only intracapsular ligament is the ligamentum teres (Fig. 5), a round ligament that connects the femoral head with the acetabulum inside of the joint capsule. The ligament is the strongest ligament in the body; it also contains blood vessels that supply the femoral head with its blood supply, although this function is less significant after childhood. The ligamentum teres also plays a role in joint stability while physically limiting the range of motion of the joint to prevent superior subluxation (dislocation of the joint) [14]. The Transverse acetabular ligament, which consists of strong flattened fibers, prevents inferior subluxation and works in conjunction with the ligamentum teres. Ligament stiffness is thought to be the primary way the body reduces the need to activate muscles to help remain upright. 


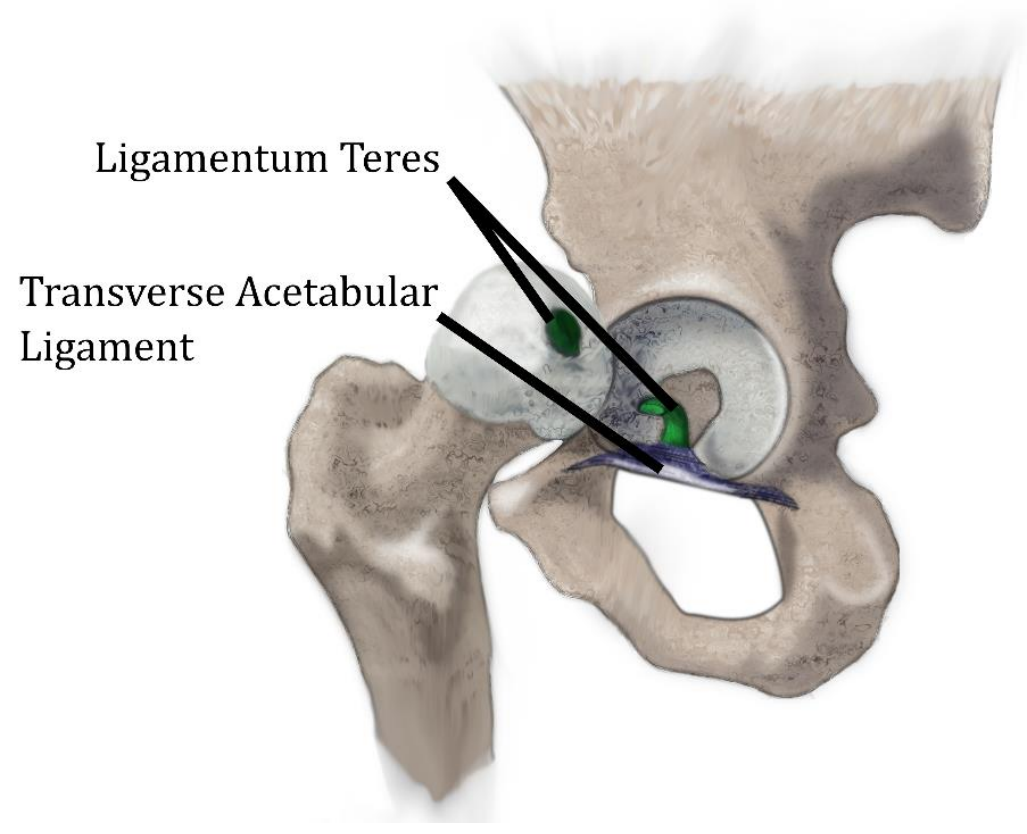

Figure 5 - Lateral view of the hip joint showing the ligamentum teres (cut into two pieces), the only intracapsular joint ligament in the body (green)

The joint capsule is filled with synovial fluid, a non-Newtonian fluid that has the constancy of the white of an egg. The purpose of the fluid is to reduce friction between the articular cartilage and joint during movement. This helps give joints their long life despite the harsh and cyclical loading conditions.

Range of motion for the hip is different for males and females; this is due to the differences in size and joint angles. To complicate this even further, age and lifestyle also have a factor on joint range of motion [15]. However, the range of motion seen in the hip joint is typically between the values listed in Table 1 . 
Table 1 - Range of motion upper and lower bounds for the human hip joint [16].

\begin{tabular}{rcc} 
& Lower bound (degrees) & Upper bound (degrees) \\
\hline Flexion & 90 & 135 \\
\hline Extension & 10 & 30 \\
\hline Adduction & 10 & 30 \\
\hline Abduction & 30 & 50 \\
\hline Internal rotation & 30 & 45 \\
\hline External rotation & 45 & 60 \\
\hline
\end{tabular}

Important to the geometry of the legs, but not specific to the hip joint itself is the quadriceps angle (q-angle). Q-angle is the angle formed by the femur and the hip relative to the ground (Fig. 6). Specifically it is the angle between a line formed by the resultant force of the quadriceps, made by connecting a point between the anterior superior iliac spine (ASIS) to the mid-point of the patella and a second line from the central patella to the tibial tubercle [17]. As this angle increases, the risk factor for patellar subluxation increases.

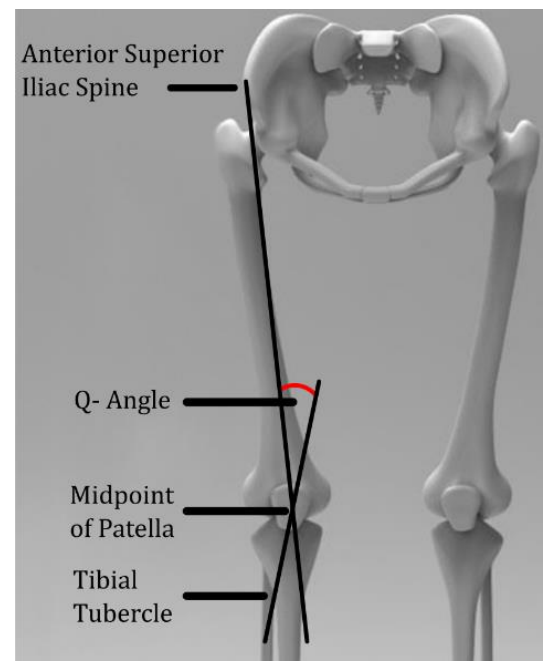

Figure 6 - Measuring points used to determine the quadriceps angle (q-angle). 
While q-angle differs between subjects and age, for males between 18 and 35 years, the average and healthy q-angle is considered $13.5 \pm 4.5$ degrees. For females, the q-angle is larger, $18.1 \pm 4.5$ degrees is considered the healthy range for subjects between 18 and 35 years $[18,19]$. Due to the cyclical loading applied to the femoral neck the qangle an important design consideration when trying to model human legs because it is a strong determiner in fracture risk [19].

\section{2 - Knee joint anatomy and physiology}

The knee joint is one of the largest and most complex joints in the body. This is attributed in part to the forces the knee experiences in normal use. Impact loads from walking or running typically exceeds 4 times bodyweight, the knee joint is responsible for mitigating some of that load.

The knee has six degrees of freedom (DOF), three rotational and three translational (Fig. 5); these degrees of freedom give the knee a complex, but needed motion for normal function. 


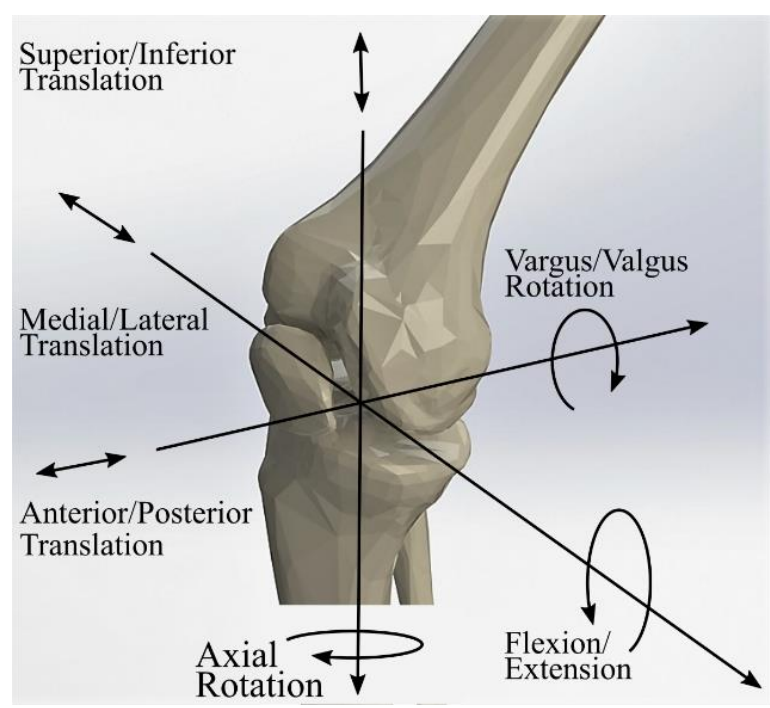

Figure 7 - The six degrees of freedom that make up the human knee

Coincidently six different ligaments provide stability for the knee (Fig. 8). These ligaments are:

1. Anterior cruciate ligament

2. Posterior cruciate ligament

3. Lateral collateral ligament

4. Medial collateral ligament

5. Popliteofibular ligament

6. Anterolateral ligament

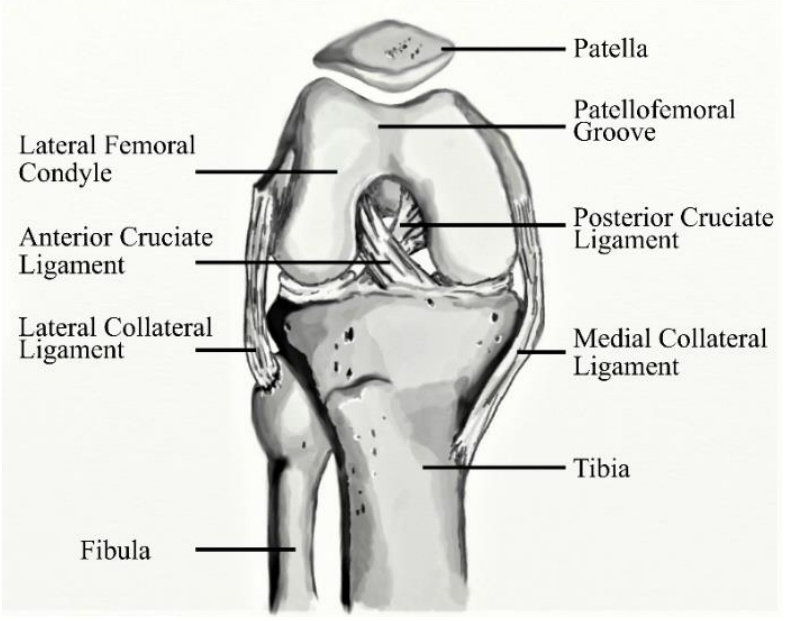

Figure 8 - The 4 main ligaments of the human knee (not included the PFL and ALL) 
The primary function of the anterior cruciate ligament (ACL) is to resist anterolateral displacement of the tibia on the femur (femur sliding backward on the tibia or the tibia sliding forward on the femur). The posterior cruciate ligament (PCL), has the primary function of resisting posterior tibial displacement (femur sliding forward on the tibia or the tibia from sliding backward on the femur), especially at 90 degrees of flexion [20].

The primary function of the lateral collateral ligament (LCL) is to resist varus displacement at 30 degrees of flexion. Serving a similar role, the medial collateral ligament (MCL) resists valgus angulation (the ligaments work together to prevent the femur from sliding side to side) [20].

The last two ligaments manage the rotational stability of the knee. The popliteofibular ligament (PFL), also known as the Posterior Lateral Corner (PLC), primary function has only been determined in the past 30 years; it resists posterior translation, varus, and external rotation [21]. It is not shown in the image above because it is posterior to the knee while the image above is an anterior view.

Lastly, the recently (re)discovered anterolateral ligament (ALL) has been shown to be an important stabilizer of internal rotation at flexion angles greater than 35 degrees. This means the ALL does not assist the ACL and PCL in stabilization during translation [22]. Because of its recent rediscovery and its location near the LCL, it too is not labeled in the diagram above. 
Because the last two of these ligaments deal with the axial rotational aspects of the knee and in robotics we want to limit the degrees of freedom for control purposes, the focus of this thesis and subsequently the joint designs, are primarily on the first four ligaments. These ligaments primarily help stabilize the knee during flexion and extension while resisting medial and lateral translation; this is the main function of the knee that we want to mimic. The additional rotational aspects are then separated from the design, and then added back in at a later time [23].

In order to minimize the force needed to deal with high loads the knee joint utilizes a changing center of rotation. As the flexion of the joint increases, the moment arm across the front of the joint increases. This mechanism keeps the joint compact and greatly reduces force requirements when compared to a static center of rotation.

The knee joint also utilizes what is called a "screw home" mechanism, called such because during the last 20 degrees of extension the tibia with respect to the femur externally rotates by roughly 10 degrees [24]. This mechanism tightens both cruciate ligaments, which lock the knee. The tibia with respect to the femur is then in a position of maximal stability. In this position, the knee requires no extra forces to stay fully extended [24].

\section{3 - Ankle and foot anatomy and physiology}

Much like the hip joint, the ankle joint is formed from several different bones that have different purposes and dimensions, but for our purposes are grouped together. For 
this reason, we are grouping the foot anatomy and physiology with the ankle joint like we did with the hip joint and pelvis.

\subsection{1 - Ankle anatomy and physiology}

The articulating surfaces of the ankle are made up of three different bones, the talus of the foot along with the tibia and the fibula, which together form a bracket shaped socket (Fig. 9). Similar to the hip joint the ankle is a synovial joint; functionally the joint acts as a hinge joint allowing for stable dorsiflexion and plantarflexion only $[25,26]$.

Eversion and inversion is a result of the movement by the subtalar joint, which is formed by the calcaneus and talus and is distant and separate from the ankle joint [27].

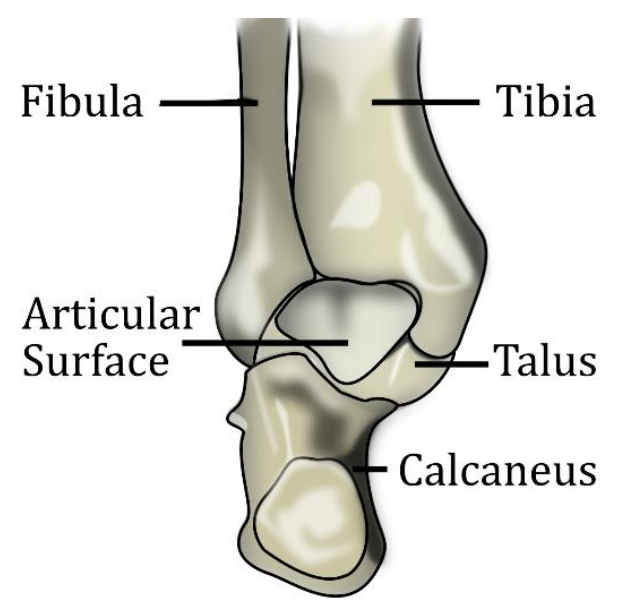

Figure 9 - The tibia and fibula form a square shaped bracket that fits around the heart-shaped articular surface of the talus

The ankle joint is held together by two main sets of ligaments both originating from a malleolus, the bony prominence on either side of the ankle. The two sets of ligaments are grouped by location medially and laterally [26]. 
While the ankle and foot has 3 degrees of freedom, modeling the ankle joint alone is typically done as a hinge, because the other degrees of freedom are created by the subtalar joint $[28,29]$. For the purposes of this thesis, we will approximate both the hip and ankle joints as ball and socket type joints. This approximation will help remove some of the instability of the ankle joint and give the joint increased range of motion.

\subsection{2 - Foot anatomy and physiology}

The foot is made up of twenty-six individual bones. The calcaneus bone is the largest, strongest, and the most posterior bone of the foot and is the attachment point for the Achilles tendon; superiorly to the calcaneus is the talus, which forms a tri-planar, but uniaxial joint with the calcaneus. The talus also serves as the loading surface for the distal end of the tibia and fibula [30].

Biomechanically, the foot behaves in two separate halves [27]. The two medial most metatarsals and phalanges move in conjunction with the talus forming a chain of interconnected motion [27]. The other three metatarsals phalanges move in conjunction with the calcaneus also forming a chain of interconnected motions [27]. Therefore, we simplify the design of the foot to just two toes instead of needing to recreate all five toes for use in mimicking the biomechanics. 


\section{4 - 3D printing with continuous fiber}

This work uses a MarkForged Mark II 3D printer [31]. The printer prints in a nylon and carbon fiber chop composite called Onyx. It also has the unique and patented ability to print with continuous fiber inlay. The printer has the ability to add continuous fiberglass, High Temperature and High Strength (HTHS) fiberglass, Kevlar or carbon fiber to the prints (Fig. 10).

With each of these fibers having its own mechanical properties, inlayed fiber is used to augment the print depending on the requirements of the part. Thus, we add strength, temperature resistance, or wear resistance by incorporating the corresponding fiber into particular layers or sections of the part.

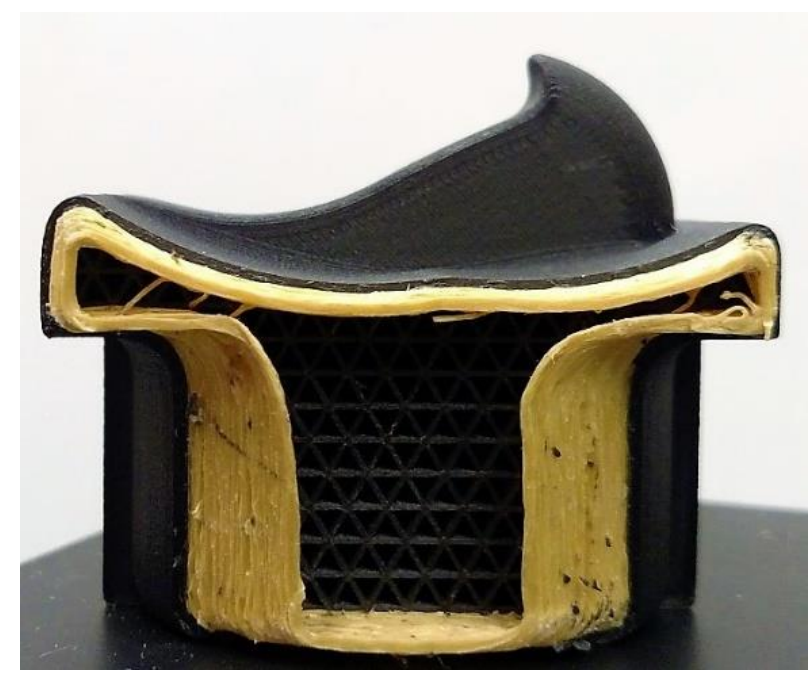

Figure 10 - Cutaway of a 3D printed part showing the continuous Kevlar fiber inlay (yellow) inside the nylon and chopped carbon fiber composite (black)

By utilizing this continuous fiber inlay, the company claims a part achieves a strength to weight ratio similar to aluminum. To back these strength claims, the company 
tested the mechanical properties of parts with continuous fiber. These properties are measured by a method similar to ASTM D790 and are shown here in table 2 [32-35].

Table 2 - Material properties for continuous fiber inlay used in the MarkForged Mark II 3D printer provided from the company. *Measured by a method similar to ASTM D790 **Two samples measured instead of five ${ }^{\# 6061-T 6 ~}$

\begin{tabular}{|c|c|c|c|c|c|c|}
\hline Property & Test Standard & Carbon & Kevlar & Fiberglass & $H S H T$ & Aluminum $^{\#}$ \\
\hline Tensile Strength (MPa) & ASTM D3039 & 700 & 610 & 590 & 600 & 276 \\
\hline Tensile Modulus (GPa) & ASTM D3039 & 54 & 27 & 21 & 21 & 68.9 \\
\hline Tensile Strain at break (\%) & ASTM D3039 & 1.5 & 2.7 & 3.8 & 3.9 & 12 \\
\hline Flexural Strength $(\mathrm{MPa})$ & ASTM D790* & 470 & 190 & 210 & 420 & 276 \\
\hline Flexural Modulus (GPa) & ASTM D790* & 51 & 26 & 22 & 21 & 68.9 \\
\hline Flexural Strain at Break (\%) & ASTM D790* & 1.2 & 2.1 & 1.1 & 2.2 & 12 \\
\hline Compressive Strength $(\mathrm{MPa})$ & ASTM D6641 & 320 & 97 & 140 & 192 & 234 \\
\hline Compressive Modulus (GPa) & ASTM D6641 & 54 & 28 & 21 & 21 & 70.3 \\
\hline Compressive Strain at break (\%) & ASTM D6641 & 0.7 & 1.5 & N/A & $\mathrm{N} / \mathrm{A}$ & \\
\hline Heat Deflection Temp $\left({ }^{\circ} \mathrm{C}\right)$ & ASTM D648 & 105 & 105 & 105 & 150 & \\
\hline Density $\left(\mathrm{g} / \mathrm{cm}^{3}\right)$ & N/A & 1.4 & 1.25 & 1.6 & 1.6 & 2.71 \\
\hline Izod Impact - Notched $(\mathrm{J} / \mathrm{m})$ & ASTM D256-10 & 958 & $1873 * *$ & 2603 & 3117 & \\
\hline
\end{tabular}




\section{Chapter 4}

\section{Methods and Materials}

This chapter presents the detailed design and implementation of hip, knee, and ankle bio-equivalent joints, and outlines their strengths as well as their limitations.

Unfortunately, the ability to measure biomechanical quantities of a joint in vivo is limited. I assume that MRI modeling of the joint provides enough biomechanical information that I am able to construct model, because the ex vivo modeling of joints removes some of the normal, or pathological conditions. From this model, I then design a mechanical joint that has properties similar to the corresponding biological joint, for instance the knee, which has a changing center of rotation and the ability to lock in the upright (standing) position.

This chapter also details the design process that was used to create the robot. I will show how I created 3D models using different medical scans and where those medical scans were obtained. Then the chapter covers each of the designed versions for each type of joint.

\section{1 - Medical scans to 3D models}

MRI scans and CT scans are similar in that they are non-invasive ways of imaging inside of the body. CT scans (or Computerized tomography) use multiple X-rays taken at 
different angles to produce cross-sectional images. MRI (Magnetic Resonance Imaging) scans use strong magnetic fields, radio waves, and field gradients to generate crosssectional images. These cross-sectional images are enhanced to show particular details of the anatomy by adding IV contrast.

CT scans are excellent at looking at bones, but do not provide a clear picture of the soft tissues without the aid of contrast. Conversely, MRI scans are excellent at detecting even slight differences in tissue and is the better choice for showing tendons and ligaments. Therefore, both scans types are necessary to develop a clear understanding of the kinematics and produce a 3D model capable of reproducing the correct motions.

\section{2 - Obtaining medical scans}

The first step was to acquire medical scans; using the open repository of medical scans provided by the software, I was able to locate several different MRI and CT scans (Fig. 11). 


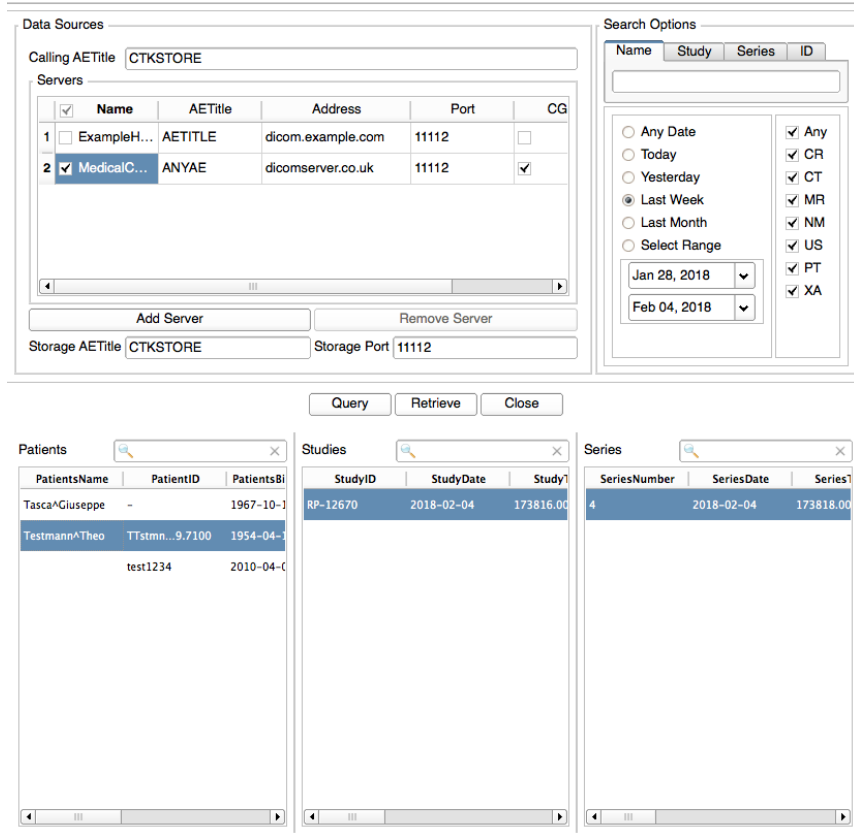

Figure 11 - DICOM library search tool layout via 3D Slicer

The DICOM files selected needed to be free from injury, had to have adequate contrast to resolve the joint structures, and need to be the same sex due to the biological differences between males and females. Furthermore, preference was given to files of patients that fell into the same age range to minimize the differences between scans. Scans were selected from adult males because the bipedal robot needed to be able handle roughly 91 kilograms (200 lbs.). Lastly, the slice thickness needed to be as small as possible to maximize the fidelity of the 3D model [36].

\section{3 - Creating a 3D model}

The process of turning medical scans to a 3D model is essentially the same for each joint; for this reason, this thesis will only go into detail about process for a single 
joint, the knee joint. The joint was selected for the next chapter because it is the most complex joint that was modeled and is the best to illustrate the process.

3D slicer was used to read the MRI and CT files.

"3D Slicer is an open source software platform for medical image informatics, image processing, and three-dimensional visualization. Built over two decades through support from the National Institutes of Health and a worldwide developer community, Slicer brings free, powerful cross-platform processing tools to physicians, researchers, and the general public."

The software was chosen because it is capable of processing a range of medical imaging data. It parses the different scans and acquire different measurements of tendons directly from the joints themselves.

To create the 3D models, the chosen medical scans in DICOM (Digital Imaging and Communications in Medicine) format were imported into the 3DSlicer software (Fig 12). 


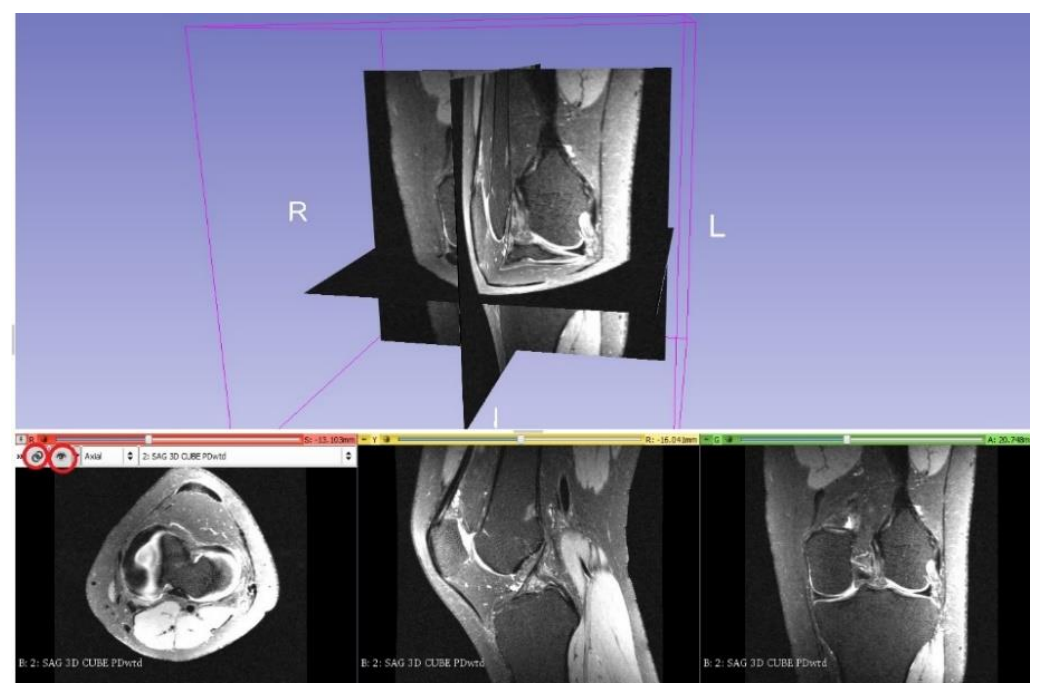

Figure 12 - DICOM files loaded into 3D slicer as viewed in the GUI

The software automatically assembles the scans into a model by organizing the scans; however, the different tissues and bones needed to be selected manually in order to create a model from the scans. The contrast of the scans was adjusted manually to provide the best intensity gradient for the section of interest (bones, tendons, and ligaments) from the surrounding tissues.

CT scans show the bone much clearer than the MRI scans while the MRI scans show the tendons and ligaments with more clarity than the CT scans (Fig 13). By utilizing these larger gradients, the software has a much easier time separating the soft tissue from bone or the ligaments and tendons from tissues. 


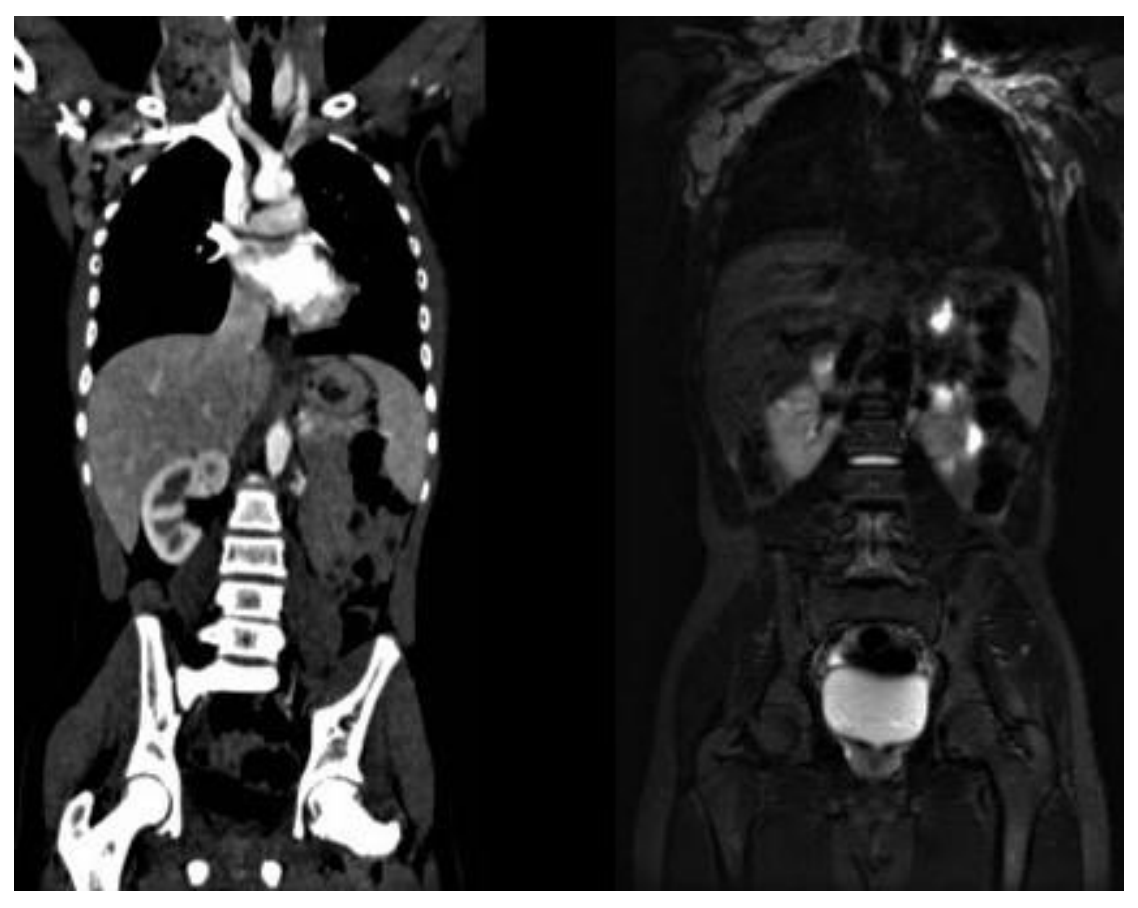

Figure 13 - Results of a CT scan (left) vs. an MRI scan (right) notice that the CT scan shows a much higher contrast for bones than the MRI scan.

The program then selects sections of each layer of the scan within the stated range (Fig. 14).

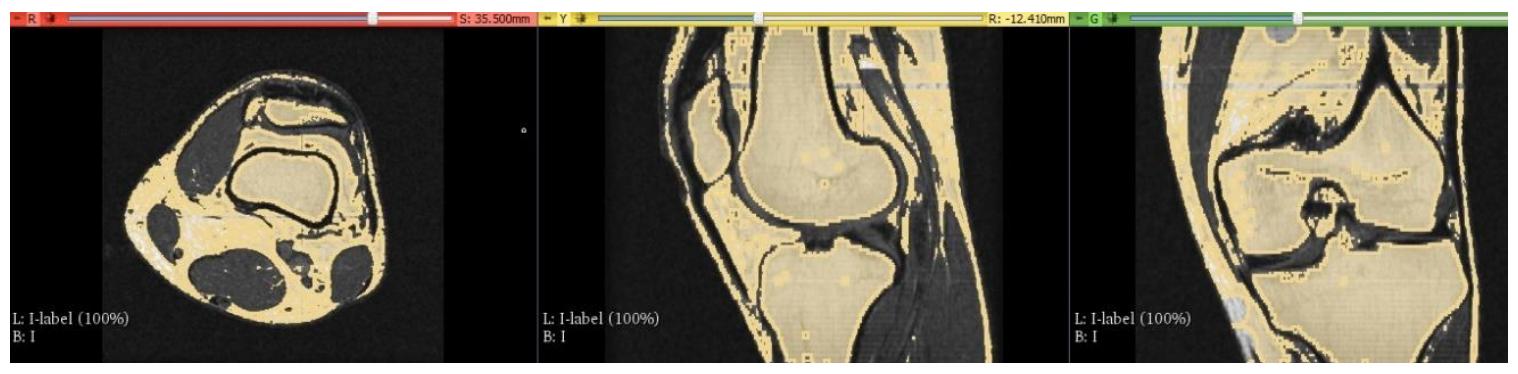

Figure 14 - Initial attempt as selecting bone exclusively using the software's selection algorithm (selection in brown)

I manually cleaned the scans once the software selected and labeled the sections as the target tissue or bone. By removing unwanted selections made by the program. This 
step verifies the software selected the correct areas of the scan and requires manually fixes any discontinuities or erroneous selections (Fig. 15).

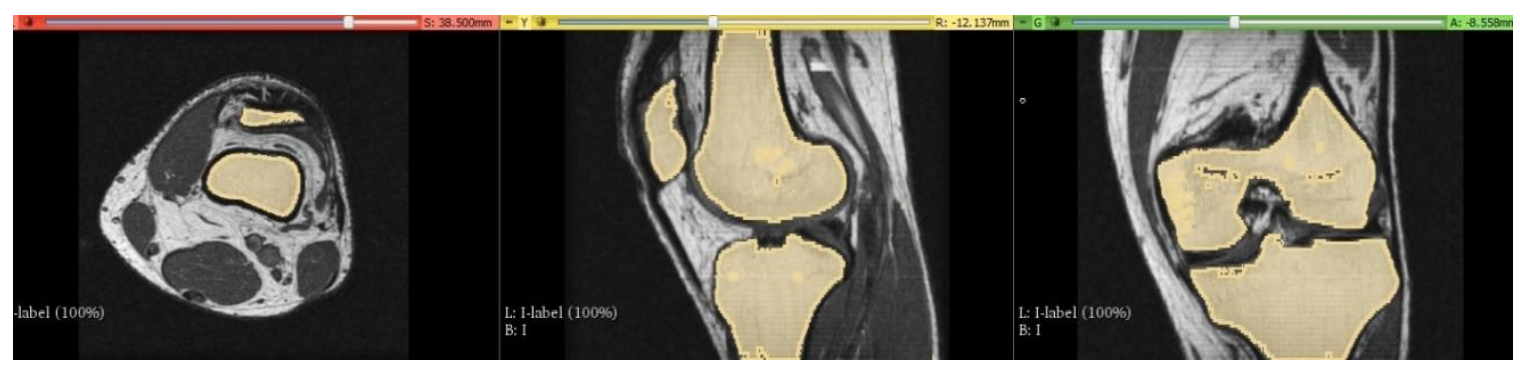

Figure 15 - Bone selection (brown) after manual cleanup and verification of selection

After manual cleaning, I converted the selections to a 3D model using the built in functionality. However, the model is not ready to be used due to how the software processes the slice thickness of the MRI and CT scans layers. Each layer is a simple extrusion to the thickness of the MRI or CT scan slice thickness, which in this case is 1 mm (Fig 16).

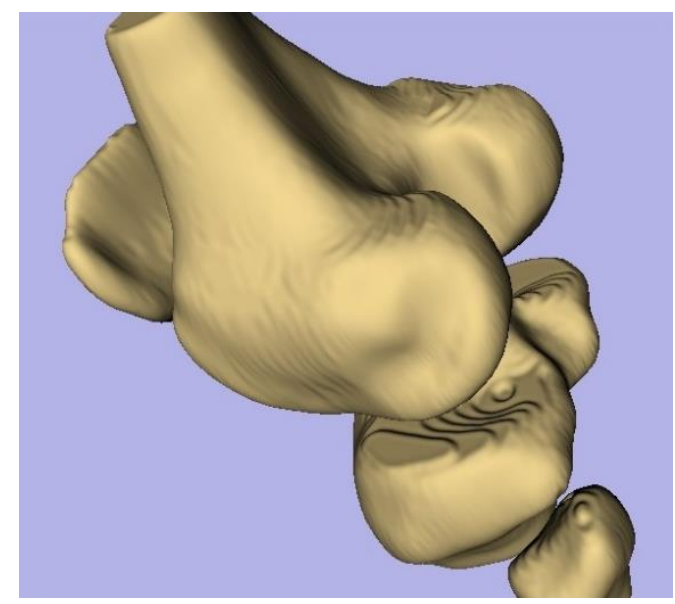

Figure 16 - Initial result from the extrude operation using 3D Slicer. This operation creates a "stepped" effect that needs to be later smoothed. 
This model must be smoothed, so the model was then exported from the 3DSlicer and into another open-source software called Mesh Mixer. This software smooths the scans by determining, then attempting to maintain, tangent continuity.

Tangent continuity occurs when the two curves share a common endpoint and are tangent to one another at that point. A high fidelity model that limits deformation from this process is created by maintaining tangent continuity preferentially over other smoothing operations (Fig 17).

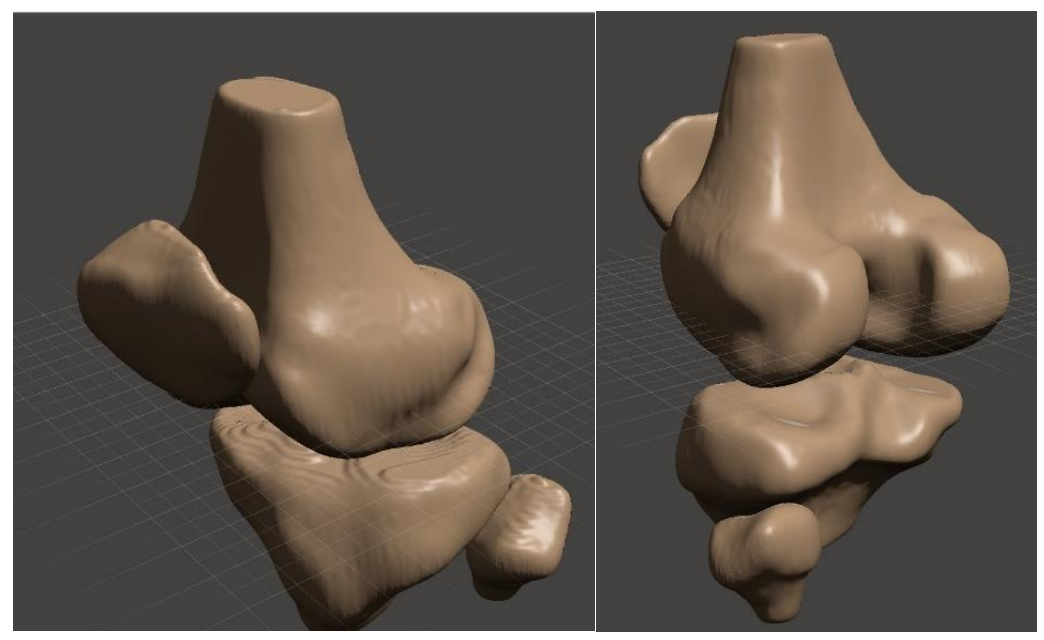

Figure 17 - (A) The 3D model from 3D Slicer loaded into Mesh Mixer and (B) after the tangent smooth operation is applied

\section{4 - Hip Joint}

The hip is made up of three different fused bones, so for the purposes of designing and building a robot this is simplified to just one. That leaves us with three separate bones, the left and right ilium, and the sacrum. 
To design the hip, CT and MRI scans of the lower extremities were used to create 3D models of the joints using an open-source software called 3D Slicer (Fig. 18). This program organizes the Digital Imaging and Communications in Medicine (DICOM) files so they are manipulatable in a $3 \mathrm{D}$ environment. The software selects specific portions of each layer, and by applying an extrusion operation corresponding to the layer thickness of the medical scan, creates a 3D model.

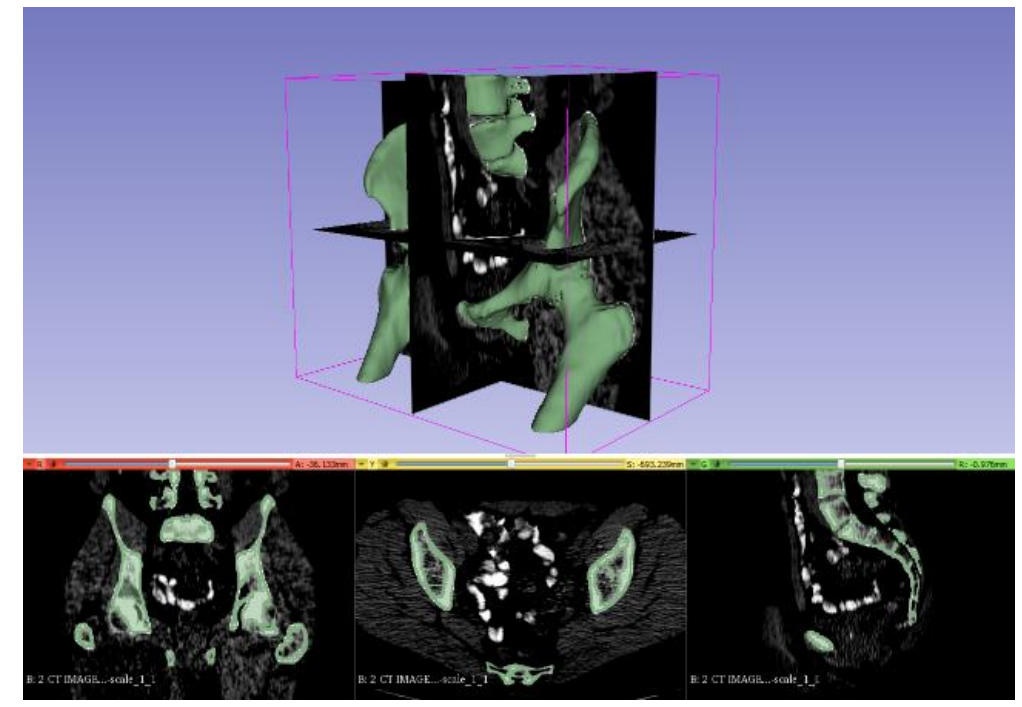

Figure 18 - Hip Model created using the extrude function in $3 D$ Slicer.

Once these layers are extruded, a tangent smooth operation is applied to the model to eliminate the stepping artifacts from the extrusion operation. Using this process, we recreate the structures from the medical scan into a high fidelity $3 \mathrm{D}$ model. From these models, the initial articulating surfaces of the designed joint are created and sized similarly to the corresponding joints in the body. The files used to create our hip model 
were obtained from an open source biomedical 3D printing community called embodi3d [37].

The hip joint has several different geometric considerations which need to be considered to maximize the life of the designed joint $[38,39]$. These geometric parameters (Fig. 19) are the femoral neck length, the narrow neck width, and the neckshaft angle. The neck of the femur is especially at risk of fracture from loading. The joint geometry in the design comes from the median values of the accepted range for an adult male and not the medical scans since these are considered the ideal values [40]. However, the overall shape of the contacting surfaces is based on the medical scan models (Fig 20).

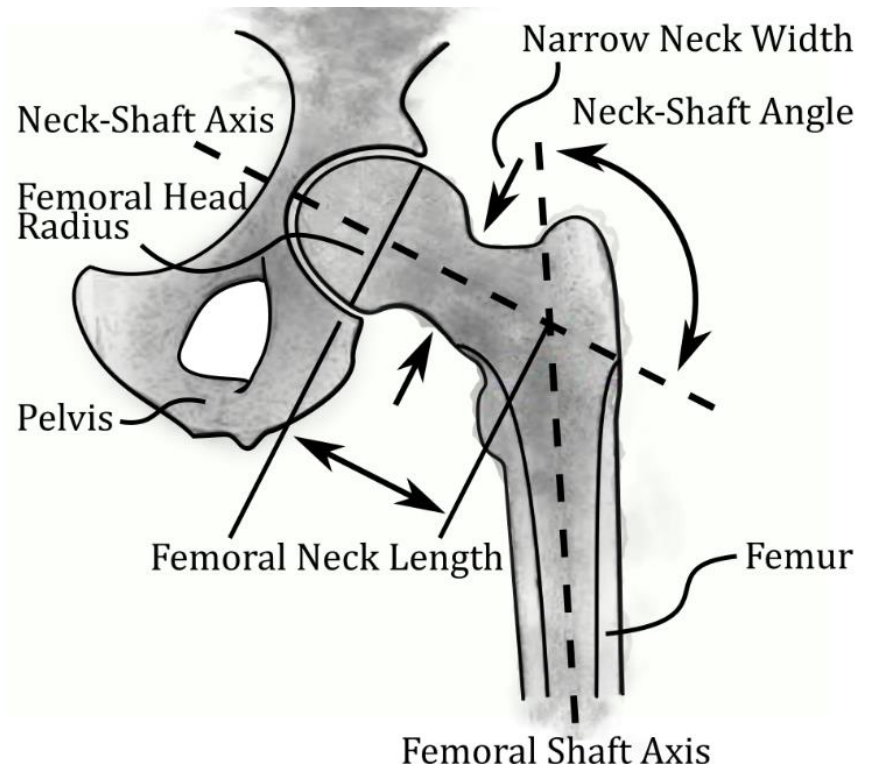

Figure 19 - Geometric considerations for designing the hip joint and femur

A way to attach the pneumatic actuator securely to each side of the joint needed to be included into the design process because the braided pneumatic actuators are going to need to resist some of the applied load. Adjustable steel hose clamps secure the actuator 
to either side of the joint; a recess on both ends of the links keeps the actuator firmly secured when tension is applied to the joint.



Figure 20 - 3D model of the designed femur

As previously mentioned, the hip is broken into three separate bones to mimic the human hip the two ilium and the sacrum (Fig 21). The parts connect to each other the same way the hip does, a spring and damping element was added between the bones using braided pneumatic actuators. These flexible connections help the hip absorb impact loading similar to how the real hip behaves when walking. 


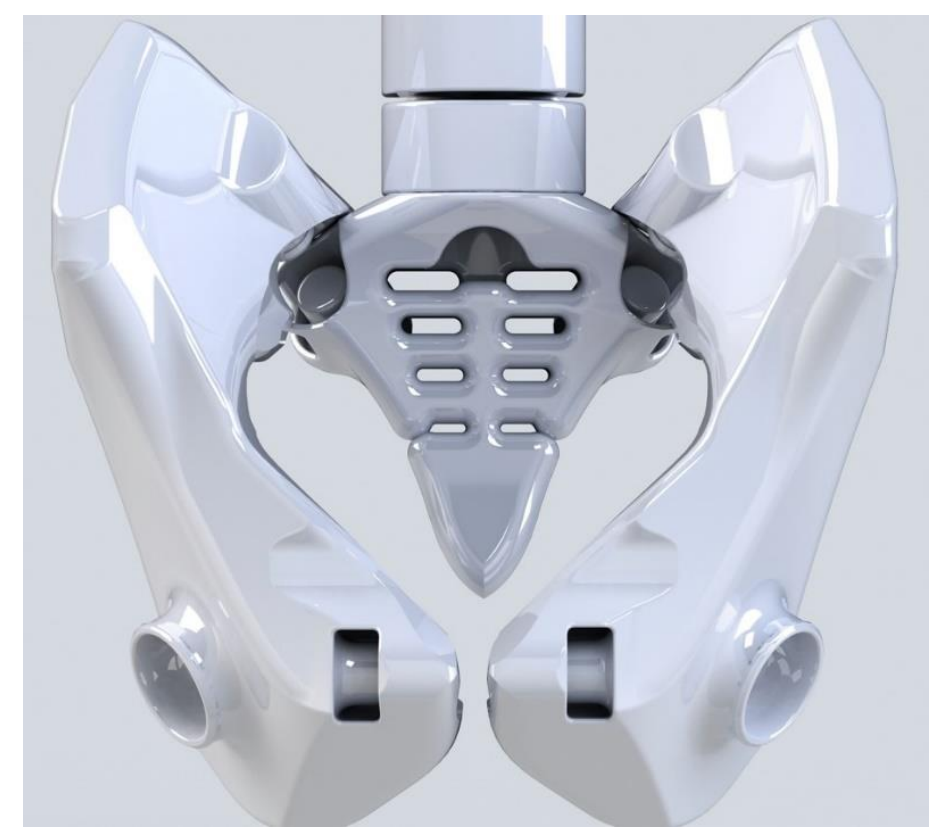

Figure 21 - Anterior view of the 3D model of the designed hip with vertebrae.

\section{5 - Knee Joint}

The knee joint is one of the most complex joints in the body. This section covers the three different versions of the joint and the design processes for each version.

\subsection{1 - Version 1}

An early solution at modeling the soft structures of the knee with rigid aluminum connections was to include an adjustable compliant element to the design. A small pneumatic actuator was selected because it matched the diameter of the human tibia. This actuator provides adjustable compliance. Furthermore, pressure in the actuator is 
adjustable to limit slip between the surfaces, the main cause of wear, and reduce impact shock as the robot moved or if the robot fell.

Machining cost was a factor so the design needed to be limited to the geometry that could be machined in house. This meant that the most complex geometry that could be created had to be machinable using a 3-axis $\mathrm{CNC}$ machine.

The LCL and MCL are located on the outer faces of the knee, so I determined machining solid metal links to act as analogs to these ligaments would be more feasible than trying to model and machine analogs of the ACL and PCL, which are located on the inner faces of the knee.

After determining which ligaments to model, I created the mechanical knee design in SolidWorks (Fig. 22). After several simulations under a variety of rotational input conditions, the proposed joint design was determined to perform similar to the biological counterpart. 


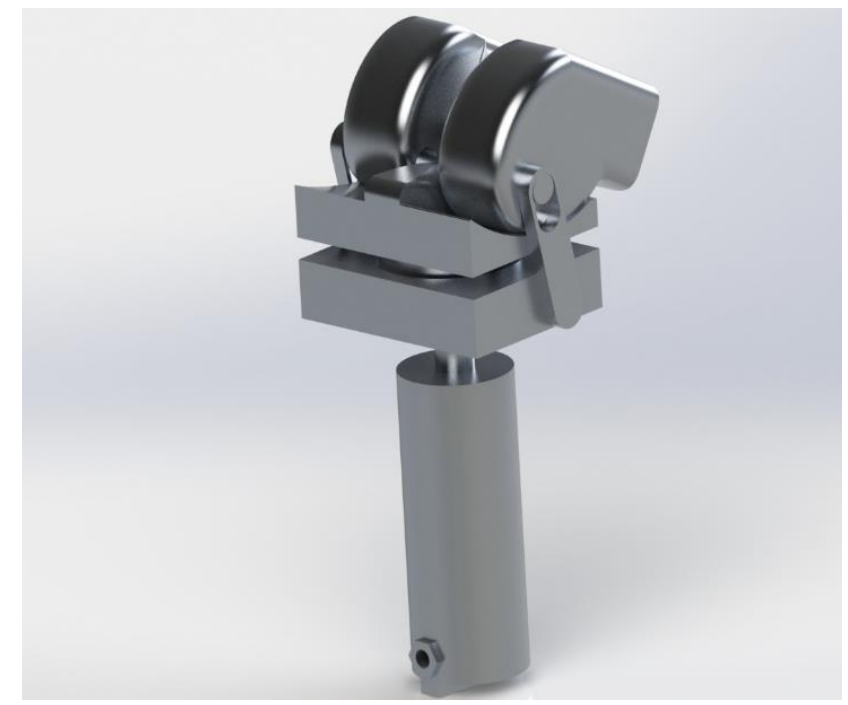

Figure $22-3 D$ model of the first version of the knee joint

To machine the part, the fibular end and tibial head (the two load bearing surfaces), were imported into a CNC program called MasterCAM (Fig. 23). Using this program, the order of cuts, the tolerances, number of passes, and finish to the design are controlled. This program also runs simulations to test the order of operations based on the sizes of the bits used and the operating conditions of the machine, which is set by the program, or by the user. 


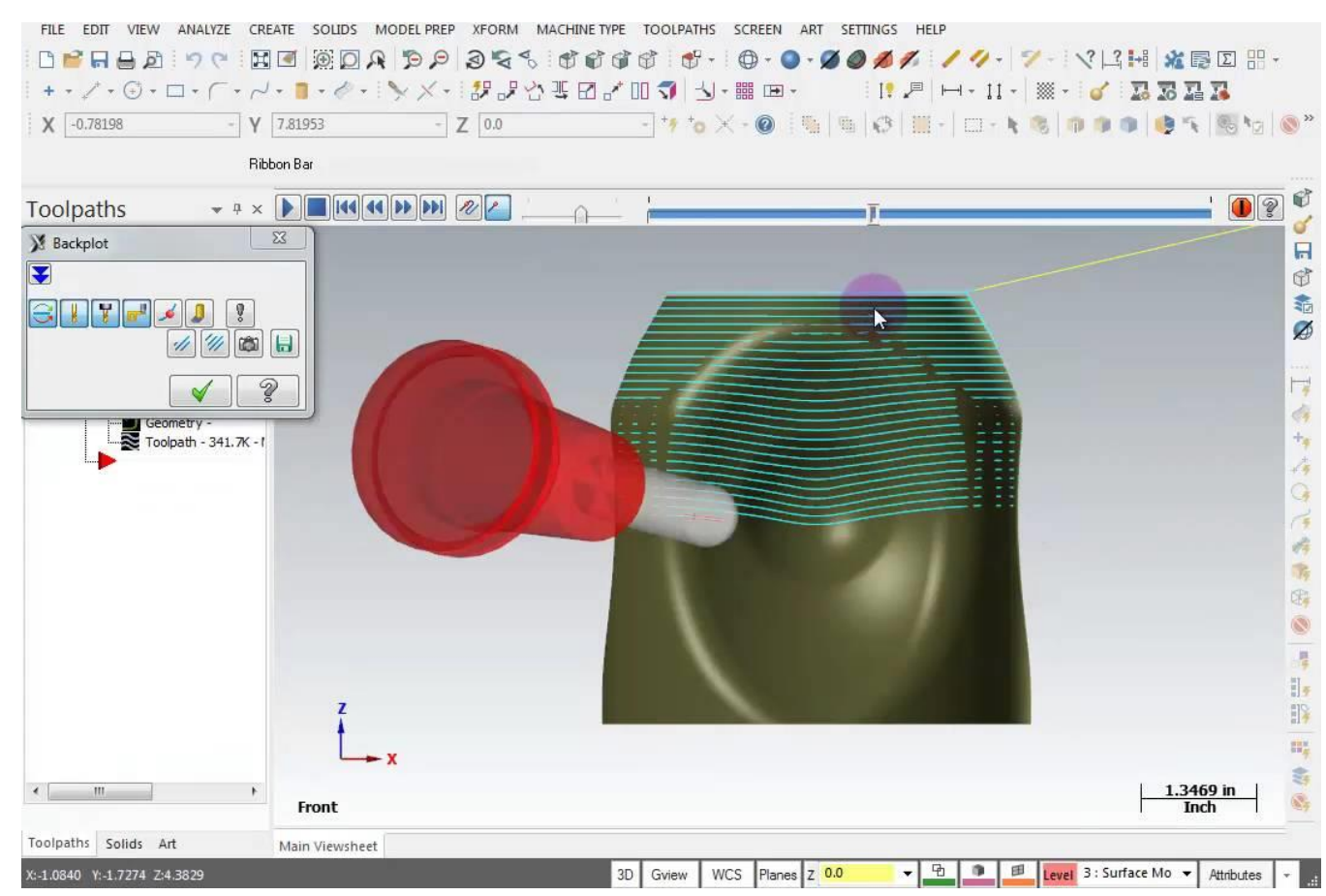

Figure 23 - MasterCAM GUI showing the tooling path.

The distal extremity of the femur and the proximal extremity of the tibia were machined using a 3-axis CNC machine (Fig. 24). This would give the best true to CAD outcome and cut the parts quicker than trying to machine each part by hand on a mill.

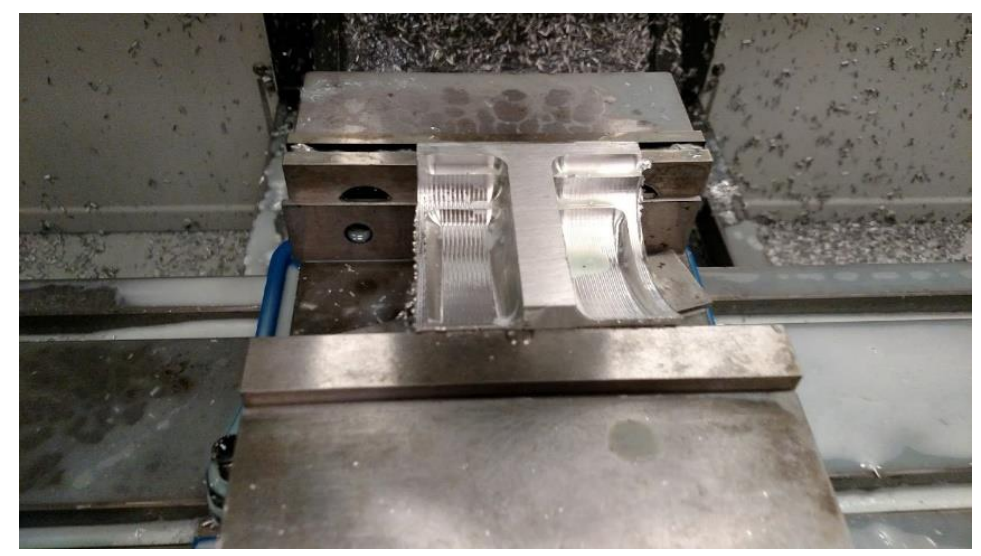

Figure 24 - Machining of the tibial head using a 3-axis CNC machine 
The rest of the parts were machined on a mill, lathe, or a 2-axis CNC machine (Fig. 25).

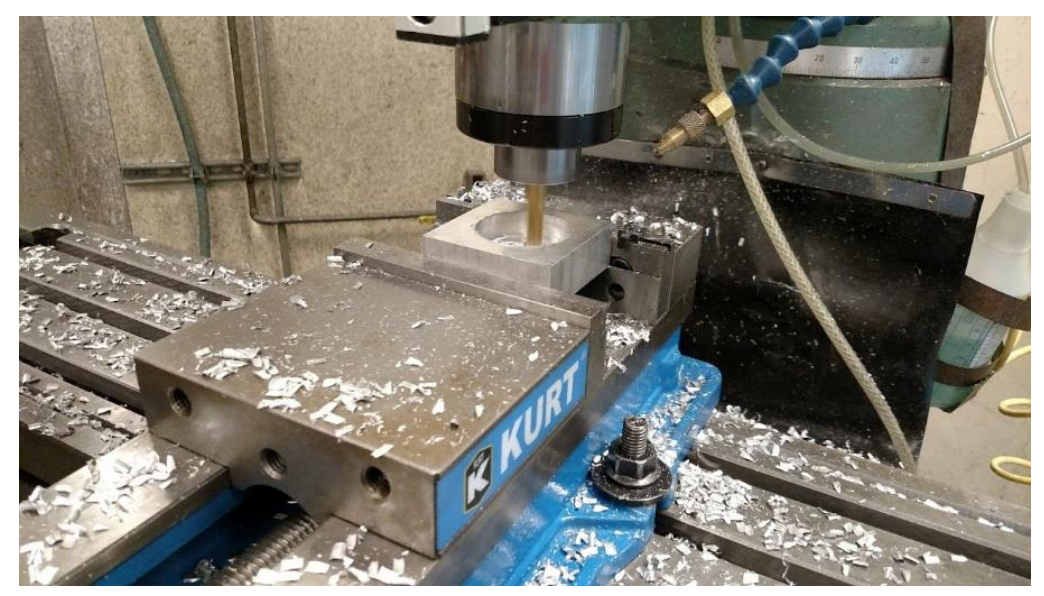

Figure 25 - Aluminum slug being machined for the tibial end using a 2-axis CNC machine.

Welding of the square tube that holds the pneumatic cylinder was done using a TIG welder. Welding of the components was done prior to assembly (Fig. 26). Between setup, takedown, clean up, and waiting to access the 3-axis $\mathrm{CNC}$ machine, the fabrication process took roughly 6 weeks from start to finish.

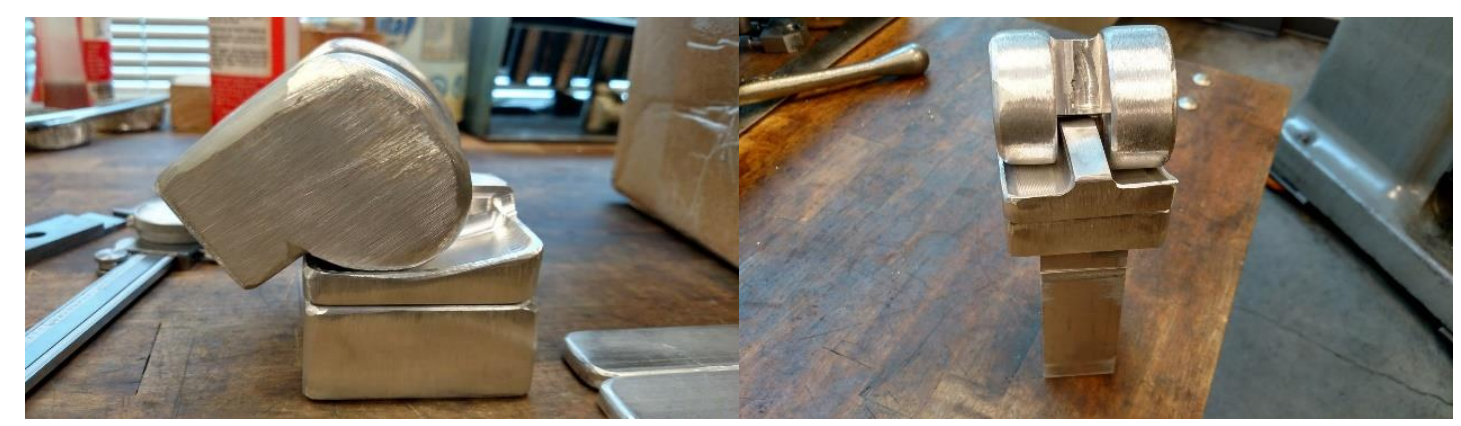

Figure 26 - Initial completion of the knee joint prior to welding 


\subsection{2 - Version 2}

Having access to new equipment, the MarkForged 3D printer, the primary goal of the second joint design was to address problems with the first design. The second goal was to determine the abilities of the new technology and to evaluate the veracity of the claims regarding material properties. Significant changes were not made to the design.

However, a few key changes were made to the 3D model to make it more suitable for $3 \mathrm{D}$ printing. The lateral and medial faces of the femoral portion of the design were hollowed to lighten the design (Fig. 27). This cut the amount of material needed, shortened the time to print, and did not affect the strength of the design. The other change was to the LCL and MCL connections. The connections were modified for added strength in $3 \mathrm{D}$ printing. There were no other significant changes made to the version one design. For example, the first iteration of the second knee joint used the same contact face angles as the first version of the joint.

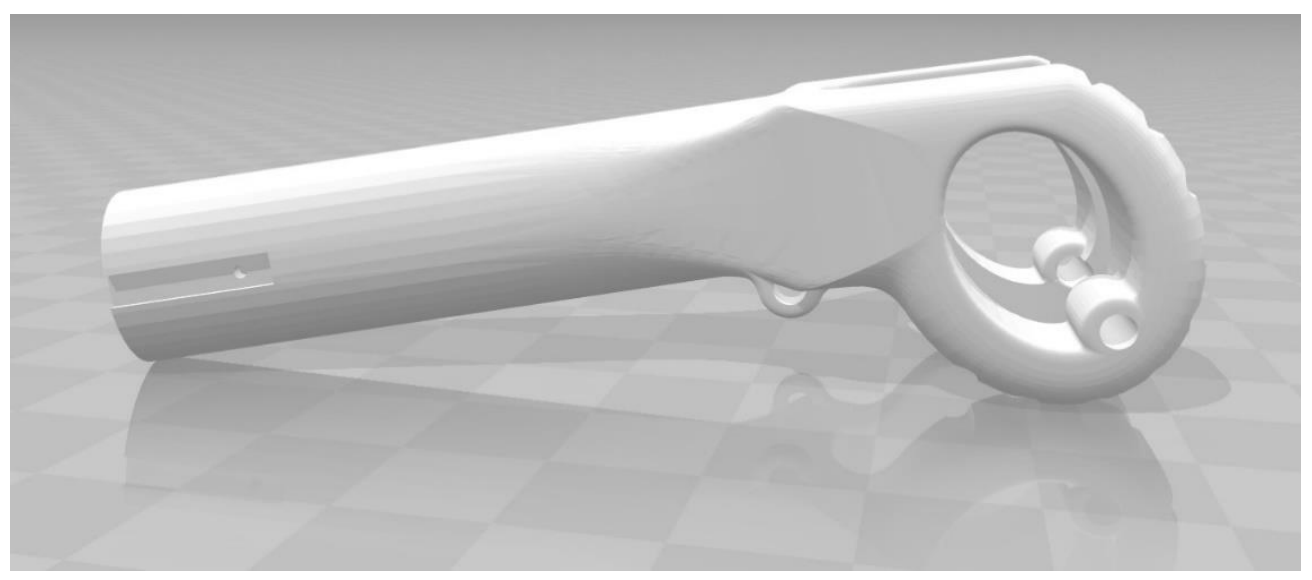

Figure 27 - Modification of the femoral end to reduce weight. 


\subsection{3 - Version 3}

However, this was changed for the second iteration of the second design. The ability to lock the joint when fully extended was added to the tibial contact face. A protrusion in the center of the tibial contact face was added. This protrusion was shaped to give the shaft that was press fit into the bearings of the femoral end a way to lock when the joint is fully extended (Fig. 28).

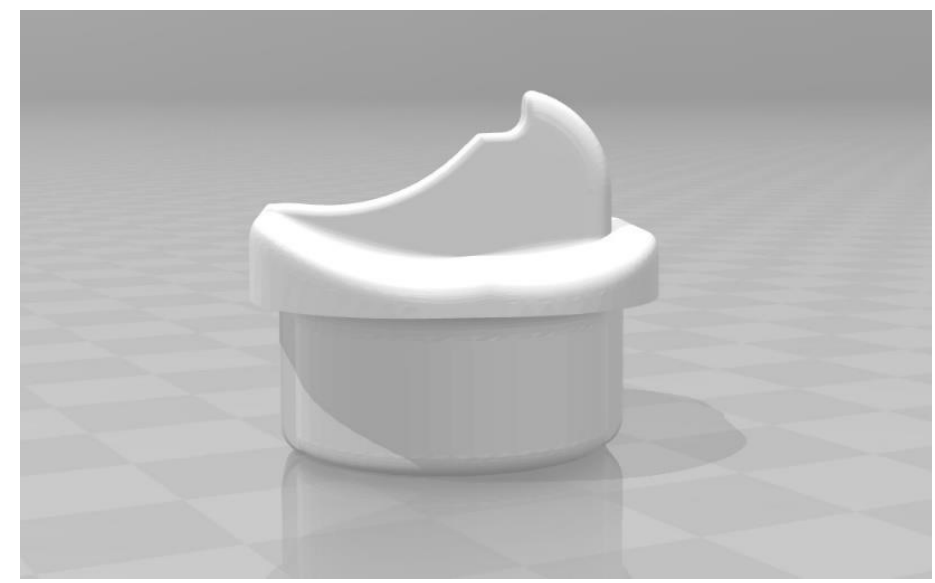

Figure 28 - Tibial head showing the addition of the screw-home mechanism

The fibular end of the joint was modified with the addition of a replaceable clipon face for the load-bearing surfaces of the fibular end of the joint (Fig. 29), which was printed using a Kevlar inlay because of the wear properties of the material. 


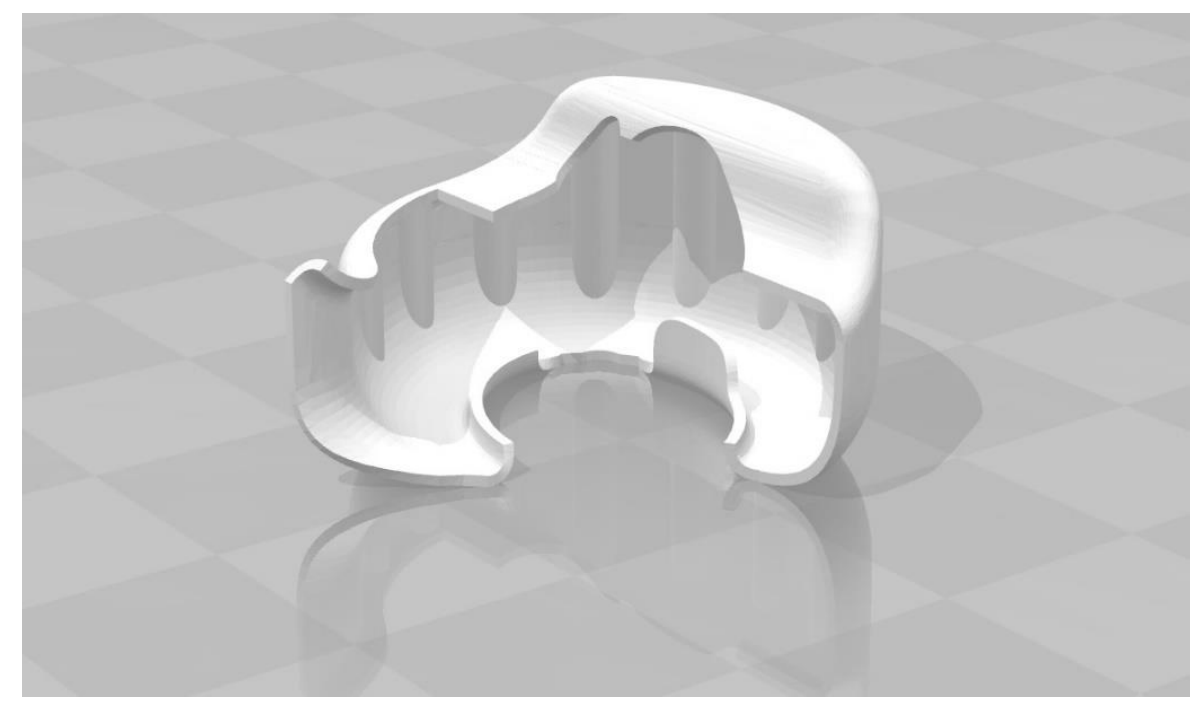

Figure 29 - 3D model of the designed clip

The fibular end was further modified to include rounded teeth that lock in with the clip-on faces. This modification provided a tight fitting connection that would not create unwanted sliding. The clip-on faces attach in such a way that they do not dislodge from the fibular end of the joint during use (Fig. 30).

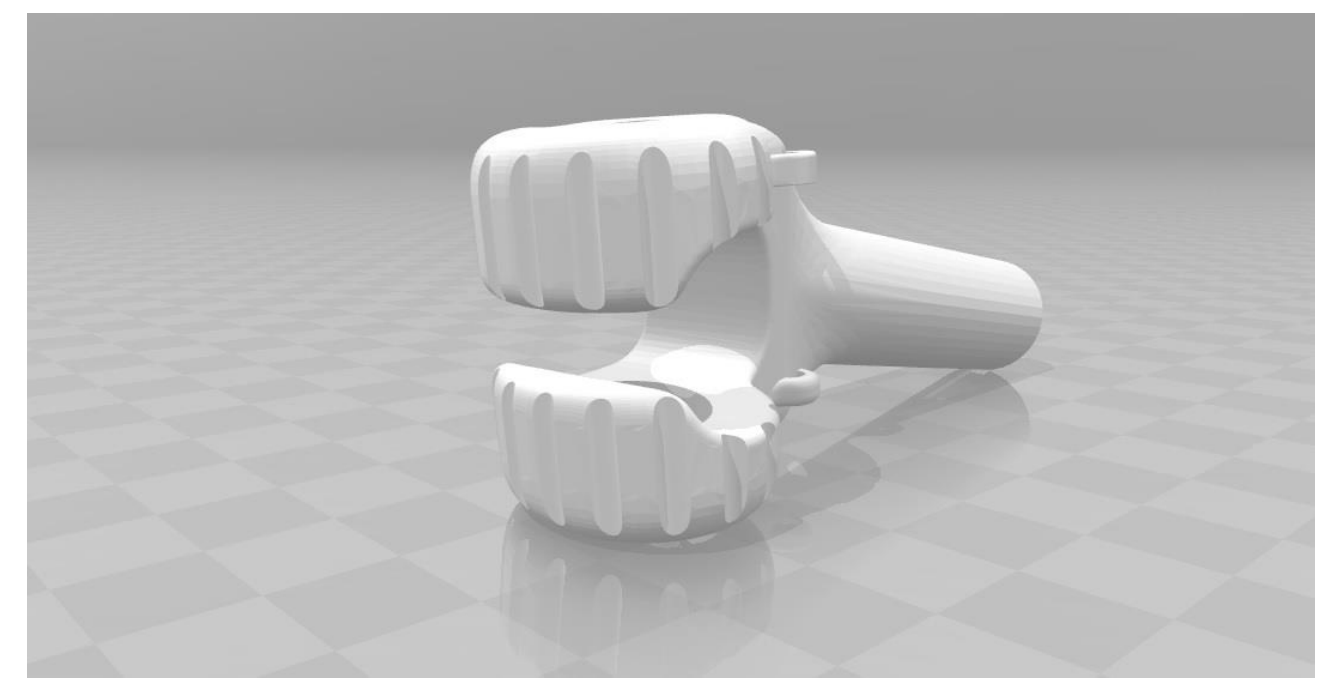

Figure 30 - 3D model showing the addition of locking surfaces on the fibular end 


\subsection{4 - Version 4}

With the additional equipment and because I was no longer constrained by the limitations of a 3-axis CNC machine, I redesigned the entire mechanism by modeling the ACL and PCL. This change meant not only could a more compact joint be created, but the geometric complexity of the joint could be increased as well.

Design of the distal end of the femur and the tibial head were done to ensure that the loading surfaces made contact throughout the entire range of motion of the joint. By doing this we redirect the forces from the linkage to the condylar surfaces, this will reduce wear on the pins and reduce the risk of catastrophic failure.

The contacting surfaces of the knee could not be copied outright, because the linkages we are using are solid and the linkages of the human knee are flexible. Instead, a new design for the tibial contact surfaces was designed to reduce sliding while still being compact. For that reason, the curve of the medial condyle, the larger of the two condyles, was chosen for both the contact surfaces of the distal end of the femur.

The tibial head was designed so that when the end of the femur is constrained to the crossed four-bar linkage it maintained contact throughout the range of motion. In order to do this, the shape of the curve created by the femur when constrained by the four-bar linkage was calculated and then used to create a 2-dimensional profile of the contact surface of the tibia. This curve was then turned into the profile of the tibial contact surface using SolidWorks. The rest of the tibial head was then designed around the movement of the linkage so that it would not interfere with the range of motion. 
Advantageously, by modeling the ACL and PCL instead of the MCL and LCL in addition to representing them by solid links, the new model without additional modifications had a simplified version of the screw home mechanism that lets the joint lock at full extension. This new locking mechanism was more robust than the one that was created for version 2 .

The analog to the screw-home mechanism is achieved using the geometry of the linkage because the design uses a solid and crossed 4-bar linkage design. Limiting the forward movement of link 1 through modification of the condylar surface of the tibial head creates a mechanical stop that prevents any additional forward movement (Fig. 31).

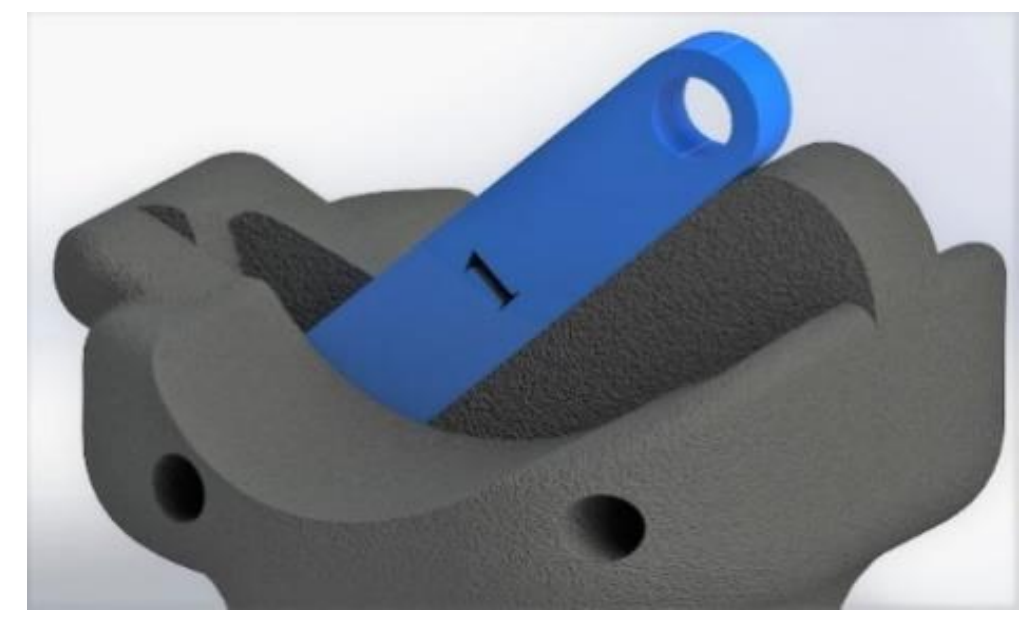

Figure 31 - The modification of the tibial head to allow for upright locking

Once the joint is in the upright position, the modification to the tibial head creates a locking mechanism such that any amount of loading force applied through the knee for example while in a standing position - cannot induce a rotation of the joint due to the forward location of the instantaneous center of rotation (ICR) at full extension. The design directs weight through the condylar surfaces of the distal end of the femur and the 
tibial head instead of the connecting links of the joint. This broad surface contact spreads out the load and reduces this source of premature wear and failure.

Once the linkage was designed, designing a patella to maximize the efficiency of the knee needed to be done. One of the first considerations for approximating the patella in the design was the contact faces. Contact needs to be maximized to reduce the wear of the surfaces. Additionally, the force vector applied by the patella to the joint should point perpendicularly to the moment arm in order to maximize the torque about the joint applied by the quadriceps muscles. The quadriceps muscles apply forces to the knee joint (Fig.32), where the resultant force $F(\theta)$ passes through the instantaneous center of rotation and is calculated by

$$
F(\theta)=2 Q * \cos \left(\frac{\theta}{2}\right)
$$

The angle $\theta$ is the angle between the quadriceps tendon and the patellar tendon. Because the ICR curve of the designed joint is flattened, the resultant force is directed perpendicularly to the moment arm and through the ICR longer than an ICR curve that was more convex. By correctly angling the patellar using Equation 1, the resultant force from the actuator is maximized through the entire extension of the joint. 


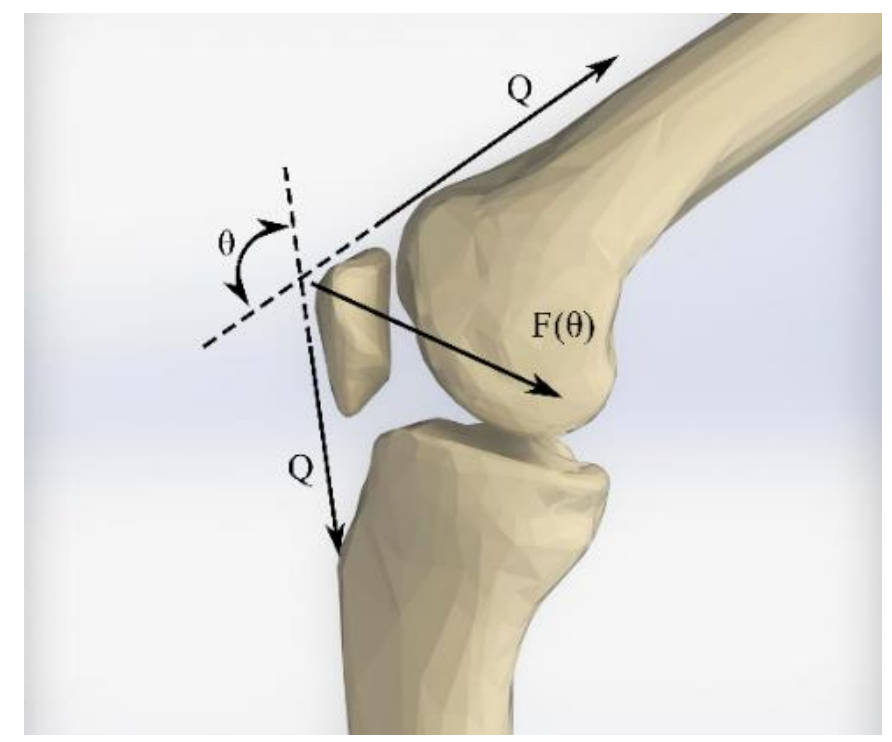

Figure 32 - Force applied to the knee through the patella as seen laterally

\section{6 - Ankle Joint and foot}

This section covers the two designs that were created for the foot, the main design consideration for the foot was that it needed to be under actuated and it needed to include the articulations that make the gait of the robot fluid (IE- the feet could not be solid). Two designs of feet were created, both of which are covered in this section.

\subsection{1 - Version 1}

The design of the foot only needed to be able to function under actuated, the first version was designed using pin joints (Fig 33) and designed using measurements of the human foot obtained from the 3D models. 




Figure 33 - 3D model of the first version of the foot and ankle

This design includes the same leaver style design the calcaneus (the bone that forms the heel) has in the foot. The protrusion (Fig. 34) adds leverage in the same manner as its biological counterpart.

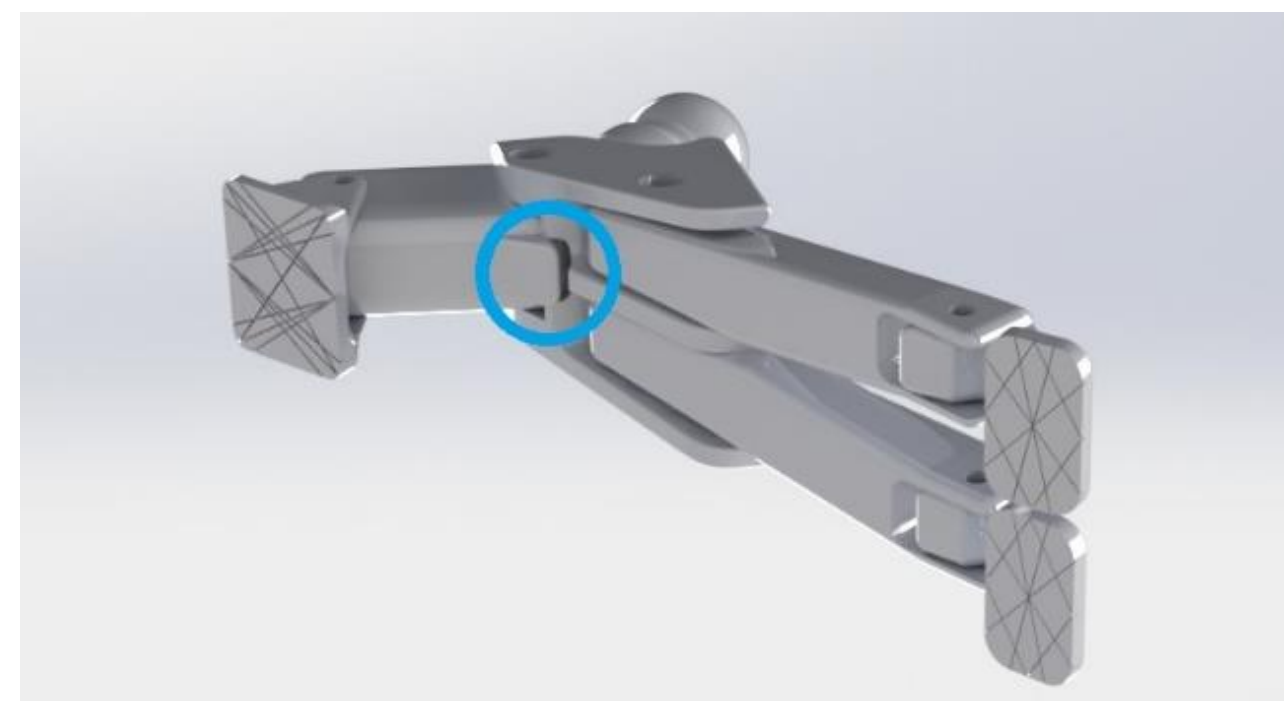

Figure $34-3 D$ rendering of the underside of the designed foot showing the addition of a lever arm to apply force to the constructed foot the same way the calcaneus does in the human foot (circled). 
A braided pneumatic actuator attaches to the underside of the foot at the rear and connects to each of the two metatarsals. This acts as an elastic element and creates the arch of the foot.

\subsection{2 - Version 2}

The second design corrects the use of pin joints in favor of using braided pneumatic actuators for attachment. The design also better incorporates the braided pneumatic actuator that is used to create the elastic element for the arch of the foot.

An extra contact point was added to the foot design so that the metatarsals of the newly designed foot make contact with the ground. This is a more natural design that gives the robot a larger contact area during the step off portion of the gait (Fig. 35).

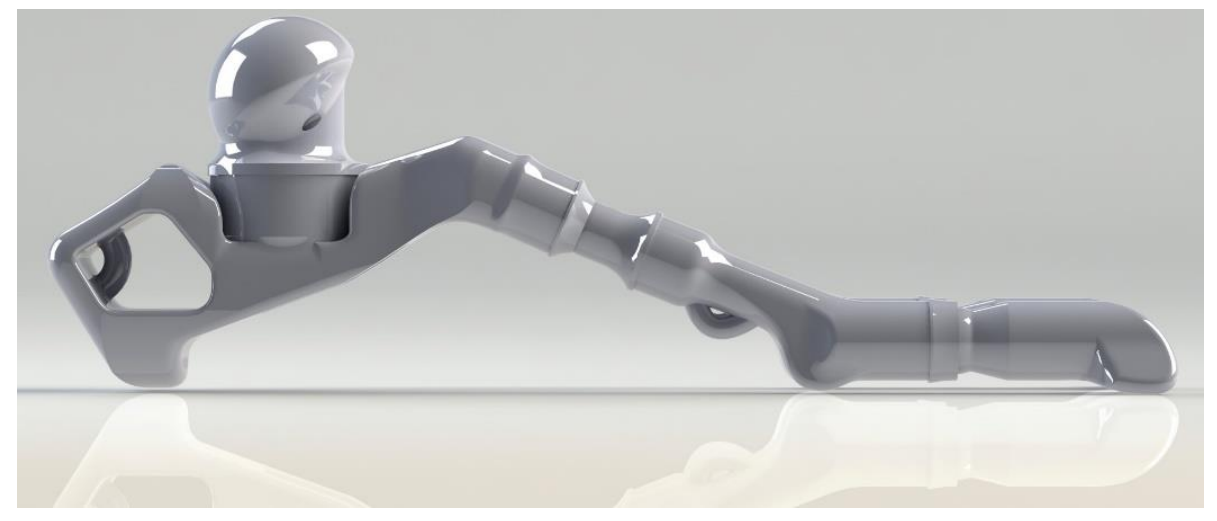

Figure 35-3D model of version 2 of the foot as seen laterally (and without the pneumatic sleeve, that attaches the joints).

Using the same pneumatic cylinder connections that are used in the hip and ankle, the pin joints were eliminated. This added damping to the foot as well as an elastic element between joints. 
The pneumatic actuator that attaches on the underside of the foot from the calcaneus to each of the metatarsals is now inside of the calcaneus (Fig. 36). Unlike the last design, the calcaneus (bone in the heel of the foot) and the talus (joint that forms the ankle) are joined together. This combined structure forms a protective enclosure for the braided pneumatic actuator used for the arch of the foot.



Figure 36 - By combining the talus and calcaneus, the pneumatic spring element used for the arch is now protected. 


\section{Chapter 5}

\section{Results}

This section covers the result of testing for each joint. It covers the performance metrics and when applicable, how the designs compare to the pin joint that they are trying to replace.

\section{1 - Hip Joint Results}

The three pieces of the pelvis are connected using braded pneumatic actuators as a spring and damping element (Fig 37).

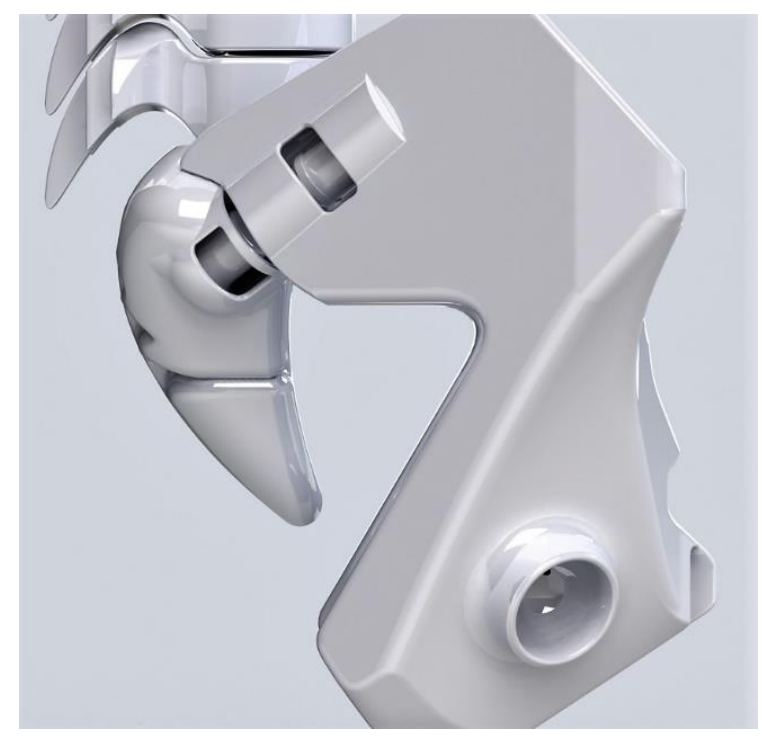

Figure 37 - Lateral view of the pelvis showing several vertebrae and better illustrating the connecting surfaces between the sacrum and ilium 
Once the hip was designed, the length of the pneumatic actuator needed to be determined in order to maximize the range of motion, because it is dependent upon the stiffness of the braided pneumatic actuator, the pneumatic actuator pressure, the joint gap, and the empty volume inside the pneumatic actuator.

The initial spacing between the two ends of the joint prior to inflation is set such that the range of motion matches closely to the range of motion of the corresponding human joint. By increasing the initial spacing of the two ends of the joint, the braided pneumatic actuator is forced to compress as the joint end comes into contact. This compression of the actuator gives the joint added range because the actuator is no longer fully extended.

Stiffness ( $\mathrm{k}$ ) for these pneumatic actuators is not constant and is a function of the length and pressure of the actuator. This stiffness function is found using a model created by Colbrunn et al. [41] by taking the derivative of actuator force with respect to length

$$
k=\frac{d F}{d L}
$$

Using a model Chou and Hannaford developed for braided pneumatic actuators [42] to determine force expressed in terms of gauge pressure $\left(\mathrm{P}_{\mathrm{g}}\right)$ and length $(\mathrm{L})$

$$
F=\frac{P g b^{2}}{4 \pi n^{2}}\left(\frac{3 L^{2}}{b^{2}}-1\right)
$$

if we differentiate (3) with respect to L (2) becomes,

$$
k=\frac{b^{2}}{4 \pi n^{2}}\left(\frac{3 L^{2}}{b^{2}}-1\right) \frac{d P_{g}}{d L}+\frac{3 P_{g} L}{2 \pi n^{2}}
$$


where $b$ is the thread length of braided sleeve and $n$ is the number of turns for a single thread in the length of the braided sleeve (Fig. 38).

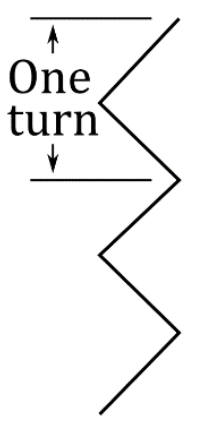

n turns

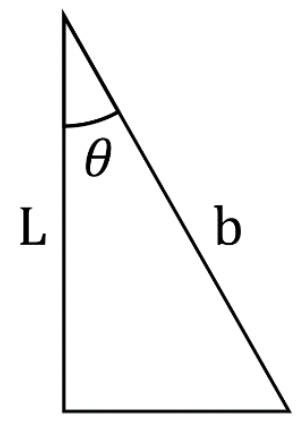

$\mathrm{n} \pi \mathrm{D}$

Figure 38 - Parameters for determining the single thread length of the braided sleeve and the number of turns for that thread.

Colbrunn et al. [41] assumes that the actuator is a membrane such that the pressure inside the actuator stays constant throughout the range of motion of the actuator. This assumption means

$$
\frac{d P_{g}}{d L} \approx 0
$$

making this assumption and applying it to (4) we now have

$$
k=\frac{3 P_{g} L}{2 \pi n^{2}}
$$

To determine radial stiffness, the beam model, where the actuator is treated as a slender member and loaded in a single plane is used. This formula relates the applied moment $(\mathrm{M})$ to the curvature of the beam $(\varphi)$, the internal pressure $(\mathrm{P})$ and the beam radius (r) such that 


$$
M=\operatorname{Pr}^{3} \frac{\frac{\pi}{2}[(\pi-\varphi)+\sin \varphi \cos \varphi]-v\left[(\pi-\varphi)^{2}-(\pi-\varphi) \sin \varphi \cos \varphi-(2 \sin \varphi)^{2}\right]}{\sin \varphi+(\pi-\varphi) \cos \varphi}
$$

where v is the Poisson's ratio of the actuator. Equation (6) is used to determine the stiffness as the joint angle changes (Fig. 39). However, this model is only valid when the joint surfaces inside the air muscle do not make contact because once the surfaces make contact we need to take into consideration the restoring forces the joint adds to the model and is no longer be assumed to be zero. The restorative force of the joint links created by the compression force of the actuator along with the added friction, increase the joint stiffness and this change needs to be taken into account.



Figure 39 - Calculated change in stiffness as it relates to joint angle at an initial pressure of 25 psi

The empty volume remaining inside the actuator has significant effects on the range of motion and stability of the joint. As a bending moment is applied to the joint, the contact surface inside the actuator moves along the ball surface, similar to the joints of the human body and is held in place by the actuator. Therefore, the empty volume inside the joint creates a mechanical limit to the amount of motion that the joint achieves. 
Table 3 shows the range of motion for the hip joints of the average adult male [43-45], the variables that impact the range of motion should be adjusted to match closely to these values while attempting to maintain maximum joint stability.

Table 3 - Range of motion of the hip joint for the average adult male

\begin{tabular}{l|ll}
\multicolumn{2}{l}{ Motion } & Range (degrees) \\
\hline Hip Joint & & $32-88$ \\
& Flexion/ Extension & $51-61$ \\
& Adduction/ Abduction & $79-99$
\end{tabular}

\section{2 - Knee Joint Results}

This section covers the results from the design of the knee joint; this includes model testing for all the versions.

\subsection{1 - Version 1}

Testing of the machined joint, (Fig. 40) met with mixed results. The simulation of the joint did not take into full consideration the effect out of plane forces would have on the joint. Due to this, along with machine tolerances - accidental damage to the prototype confounded this issue - the joint did not appear to be suitably stable when applying a combination of force and torque. This instability caused the femoral head to rotate out of the sagittal plane which was would lead to premature wear and could cause damage to the joint. 


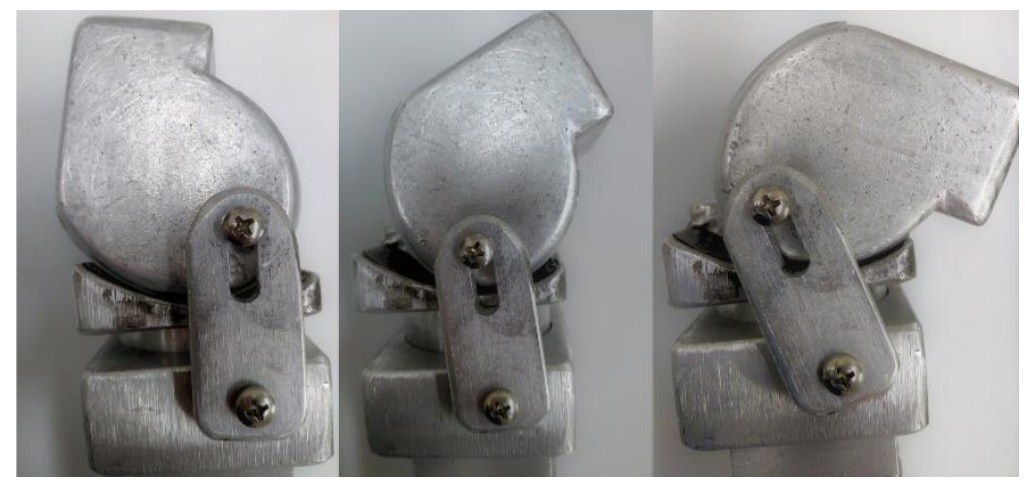

Figure 40 - Version one of the knee prototype showing how the pneumatic actuator changes as the knee rotates Figure 39 shows the assembled joint in different states of flexion

The tibia and femur bones attach to the knee using a $44.5 \mathrm{~mm}$ square tube stock. The condylar joint was created from machined aluminum stock using the MRI scans. The contact surfaces were lined with strips of UHMW plastic film, which helps reduce the coefficient of friction and gives the joint a smooth, wear resistant surface.

The design did not include the cruciate ligaments, but retained the different curved profiles of the lateral and medial femoral condylar surfaces. The intercondylar tubercules (center of tibia) have been expanded upon and used as a rail to help direct the femur in the case of high out of plane forces acting on the joint. For the prototype joint, the patellar surface has been machined in order to guide the patellar analog similarly to a biological femur.

The use of the pneumatic cylinder as an adjustable complaint member worked as expected and added stability to the joint, but also created an internal joint torque pointing towards 45 degrees from full extension. 
As previously mentioned the human knee has a screw home mechanism that locks the knee when in the upright position. The design also needed to be redesigned to let the joint lock in the upright position.

No testing of the joint was done and instead it was redesigned to better deal with out of plane forces. Additionally, the weight of the joint needed to be reduced, so other material options needed to be explored.

\subsection{2 - Version 2}

The second version design was 3D printed instead of machined. The new design underwent modifications to fix the instability of the first joint design and decrease the weight (Fig. 41).

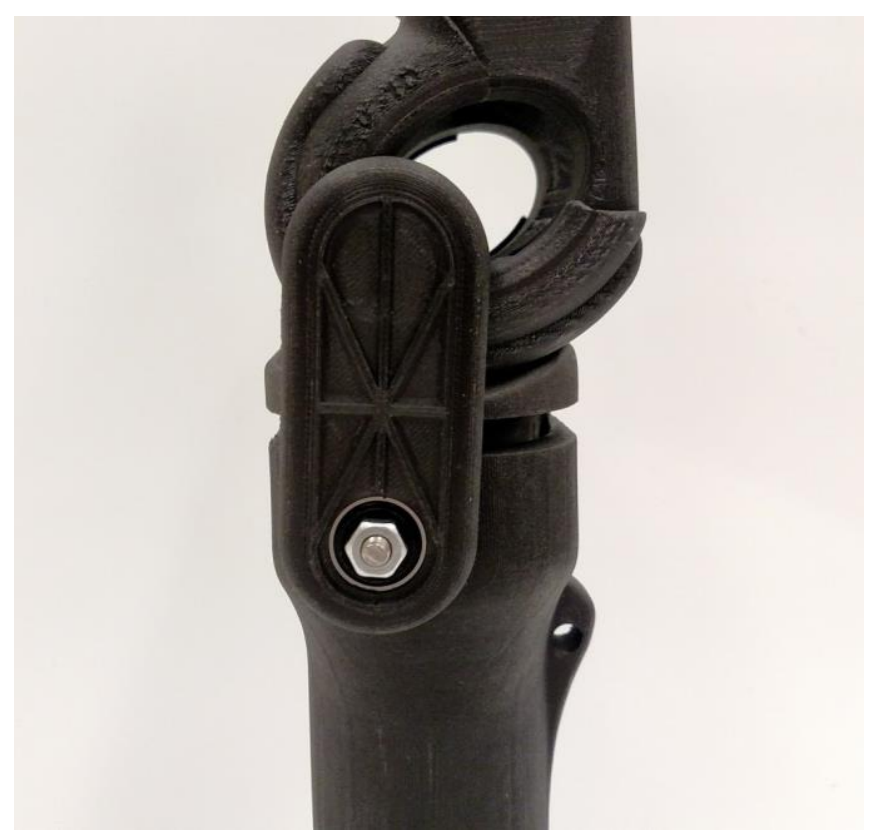

Figure 41 - Fully assembled second version of the knee joint with the cylinder inflated. 
The design has a clip-on loadbearing face for the distal end of the femur. This clip-on end helps reduce cost by giving the option of replacing only the loadbearing surface (Fig. 42).

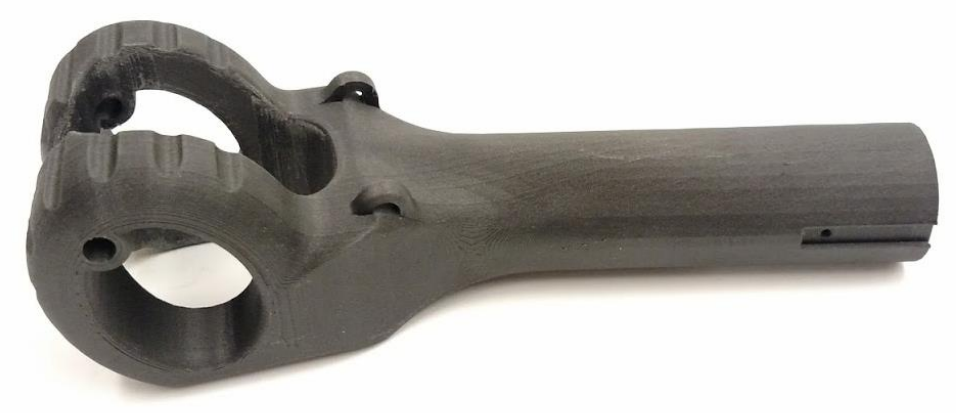

Figure $42-3 D$ printed femoral end with the clips removed from the end

These grooves help keep contact between the clip-on face and the femoral end to reduce unwanted movement between the two components (Fig 43).

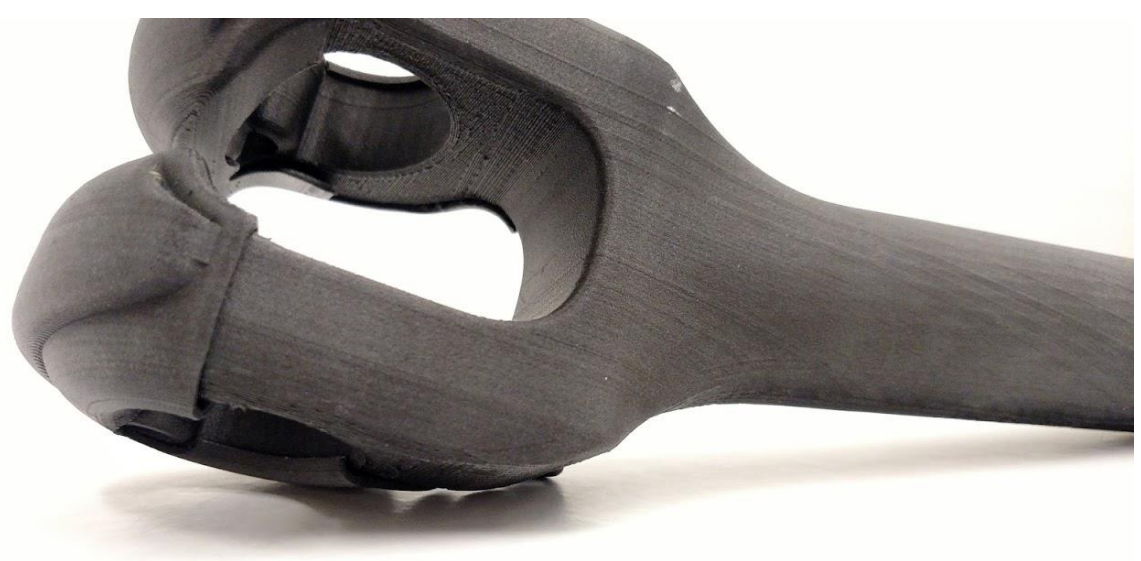

Figure 43 -3D printed femoral end with the clips inlayed with Kevlar added back into the femoral end 
The design includes a mechanism for locking the joint in the upright position, similar to the screw home mechanism. This addition eliminated the instability the first design had when torque was applied perpendicularly to the joint.

Several design changes to the screw home mechanism had to be made in order for it to function properly; these modifications were made to the center protrusion and consisted of changes in the geometry of the locking portion itself, where the metal bar would rest (Fig. 44).

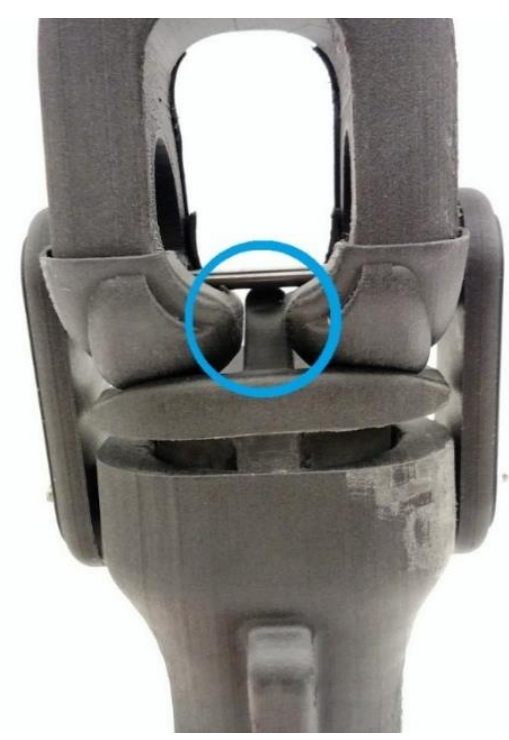

Figure 44 - Second version of the 3D printed knee showing locking mechanism (circled)

By increasing the width of the LCL and MCL analog connections, the design reduced the deflection from out of plane forces significantly. This design change also created enough room to use roller bearings; this smoothed the joint movement, reduced the friction, and therefore likely increased the life of the joint when compared to the Version 1 metal-on-metal design. 
This joint underwent a series of tests to determine the length of the torque arm, range of motion, and center of rotation (Fig 45).

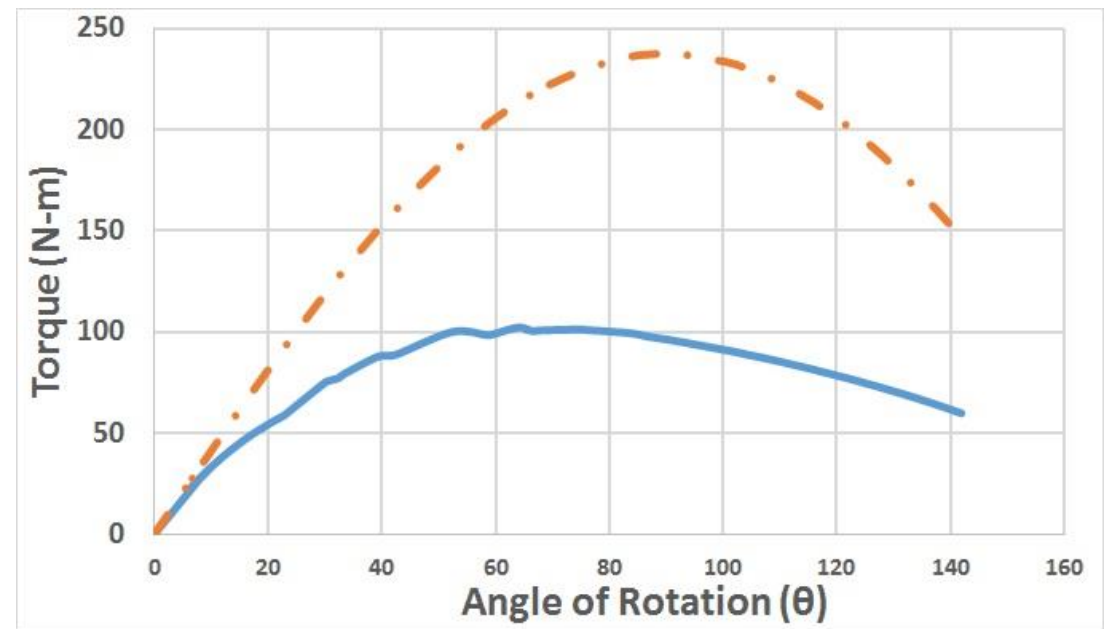

Figure 45 - Theoretical torque requirement to move a 46-kilogram load over the angle of rotation (blue solid line) vs the torque required for a pin joint (red dashed line)

The joint has a theoretical maximum torque requirement of $100 \mathrm{~N}-\mathrm{m}$, which is less than half the torque required to move a pin joint with the same weight.

The sliding to rolling ratio is an important parameter for the assessment of the joint since it estimates the amount of wear on the joint, it was necessary to determine the ratio for the proposed condylar joint [46]. This is calculated by modeling the contacting surfaces of the tibia and femur and the 3D model to create a motion study using SolidWorks software.

Rigid body kinematics is applied to determine the sliding to rolling ratio. The ratio is calculated as the difference between the arc lengths on each contacting divided by the arc length of the moving link, or: 


$$
\chi(\theta)=\frac{\Delta L_{\text {tibia }}(\theta)-\Delta L_{f e m u r}(\theta)}{\Delta L_{-} t i b i a(\theta)}
$$

where

$$
\begin{gathered}
\Delta L_{\text {tibia }}(\theta)=C_{\text {tibia final }}(\theta)-C_{\text {tibia inital }}(\theta) \\
\Delta L_{\text {femur }}(\theta)=C_{\text {femur final }}(\theta)-C_{\text {femur inital }}(\theta)
\end{gathered}
$$

are the corresponding incremental differences of the contact arc lengths (Fig. 46).

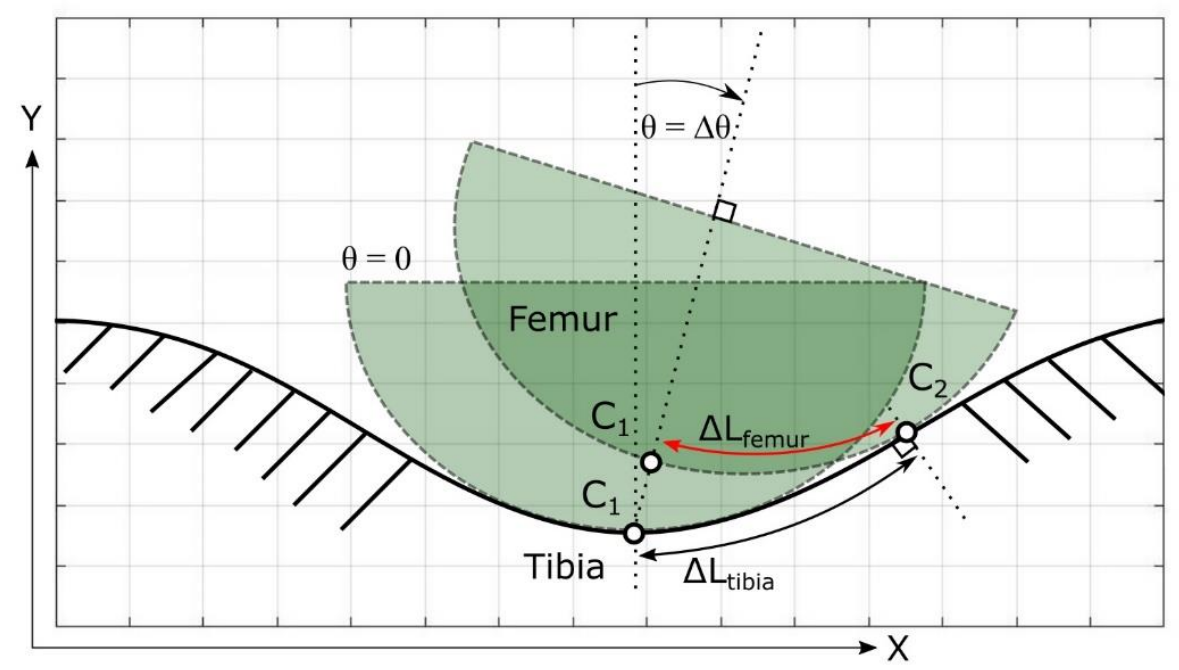

Figure 46 - Kinematics of sliding to rolling ratio from initial contact point $\mathrm{Cl}$ to $\mathrm{C} 2$

A sliding ratio of zero indicates pure rolling and a ratio of one signifies pure sliding. If the ratio is between zero and one, the movement is characterized as partial sliding and rolling. For example, a sliding-rolling ratio of 0.7 resulted in $70 \%$ sliding and only $30 \%$ rolling. If the ratio is positive, then the slip of the femur is higher than the tibia. If the sign is negative, then the tibia has higher slip when compared to the femur. 
It is desirable to have the slip as close to zero as possible to minimize the wear of the joint. The sliding-rolling ratio calculation was done over the range of the entire range of motion of the prototype joint from Eq. (8). SolidWorks simulations were then done to determine the sliding-rolling ratio of the proposed knee design with an incremental angle ( $\theta$ ) of 0.75 degrees (Fig. 47).

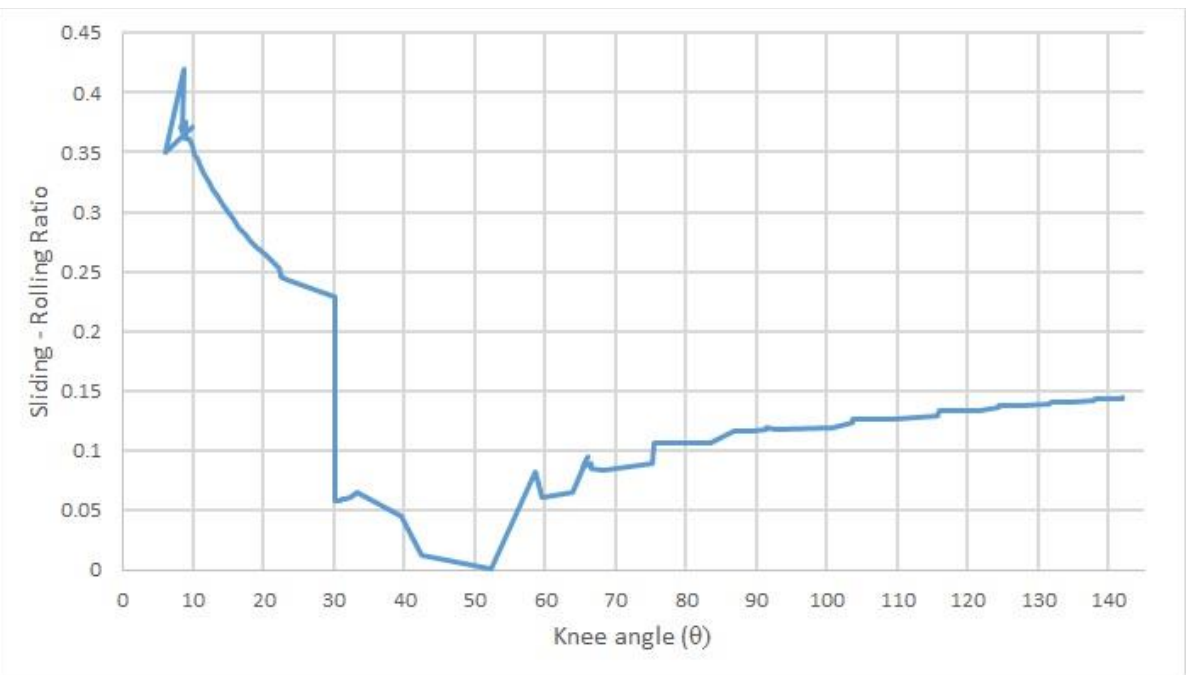

Figure 47 - Sliding and rolling ratio of the proposed joint. Note, the initially high sliding ratio settles because the model settles prior to the start of the rotation.

Our model suggests a sliding-rolling ratio of 0.159 , but if we disregard the initial settling of the 3D simulation, the sliding-rolling ratio drops to 0.126 . Due to the complex nature of the human knee, several attempts have been made to analytically model the sliding-rolling ratio [46, 47]. However, these models make several simplifying assumptions that do not accurately characterize the movement of the joint. We cannot confidently estimate the sliding ratio of the human knee joint for comparison, but we expect that the sliding-rolling ratio of our model to be similar to that of the human knee 
(0.3 - 0.5) [47]. It should also be noted that the sliding-rolling ratio of the human knee changes with the change of flexion angle, as does our model plotted in Fig 46.

The proposed joints' mechanical advantage directly influences the selection of actuators that are used. To calculate the mechanical advantage, the length of the radius $\operatorname{arm} r(\theta)$ needs first to be determined. Using the radius arm the mechanical advantage of the joint is determined (Fig. 48). The mechanical advantage (MA) is calculated by the following

$$
M A=r(\theta) / C
$$

where $\mathrm{C}$ is the distance to the center of the joint.

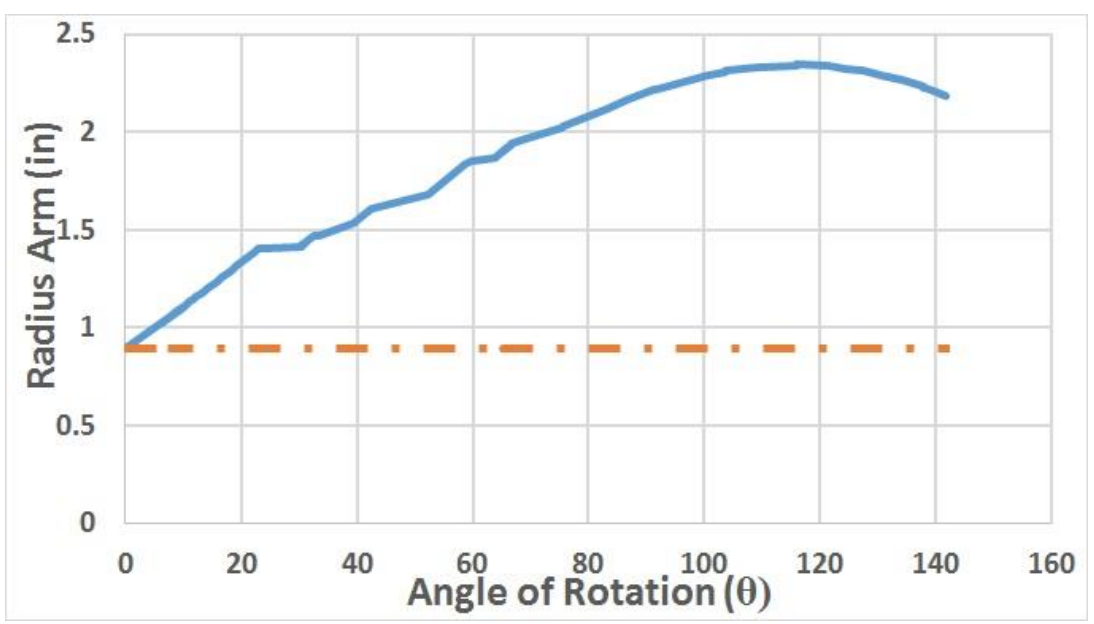

Figure 48 - Radius arm $r(\theta)$ changing as a function of the angle of rotation $\theta$ plotted against the radius arm of a pin joint (red-hashed line) 


\subsection{3 - Version 3}

Initial prototyping of the joint showed that out of plane forces has a significant effect on the joint when it reached full flexion. To better deal with this, several modifications to the tibial head were made as initial prototype testing went on (Fig. 49).

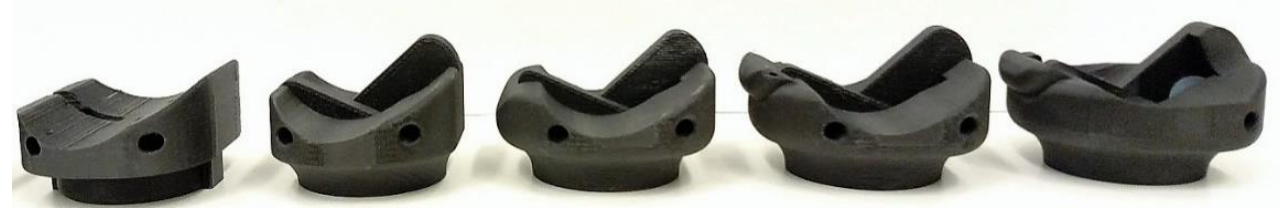

Figure 49 - Tibial head changes as the prototype testing progressed

A locking face was added posteriorly to the tibial head (Fig. 50) to eliminate deflection from out of plane forces. This addition was designed specifically to fit into the slot on the distal end of the femur. This had two positive changes to the joint design; first, it becomes the load bearing surfaces for the joint, keeping perpendicular forces from being loaded onto the ACL and PCL of joint. Secondly, it increases the moment arm of the joint to the full length of the tibial head. 




Figure 50 - Tibial head seen laterally (A) prior to modification, (B) post modification, change highlighted in red circle, and $(C)$ tibial head locked with the distal end of the femur for reference.

The constructed joint (Fig. 51) will be tested later to verify the theoretical results.

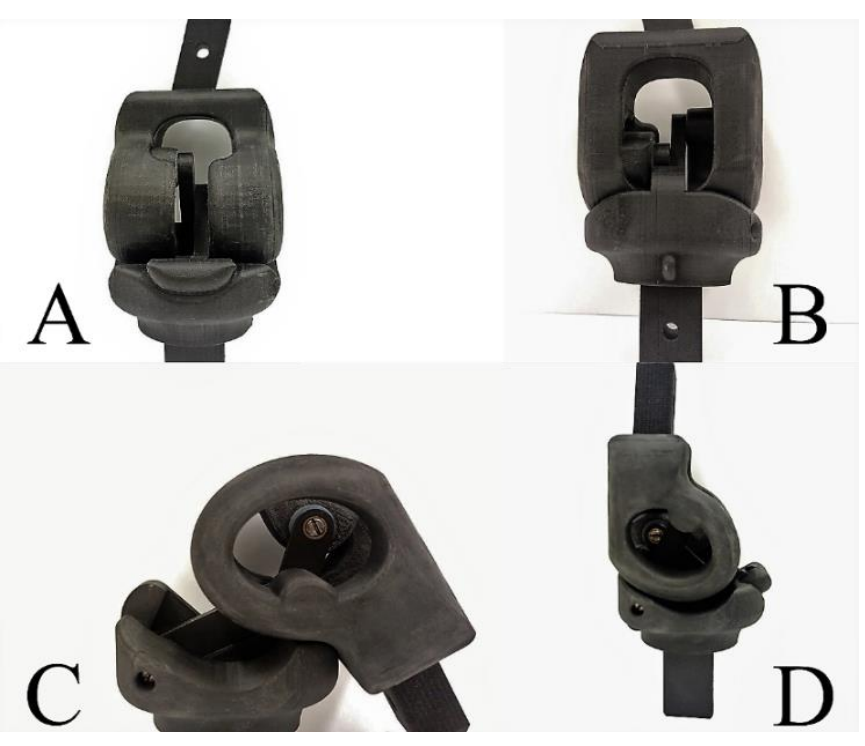

Figure 51 - Constructed joint (A) rear view, (B) front view, (C) full flexion, and (D) full extension

Metal fasteners were placed directly into the print so that metal shafts and lubricated sleeve bearings could be added to reduce friction at the joint connections. By 
adding the fasteners directly into the print, a clamping force is not required to keep the link on the shaft.

Figure 52 shows the linkage configuration used in the construction as well as the link designation, angle designation, and coordinate system for the following equations.

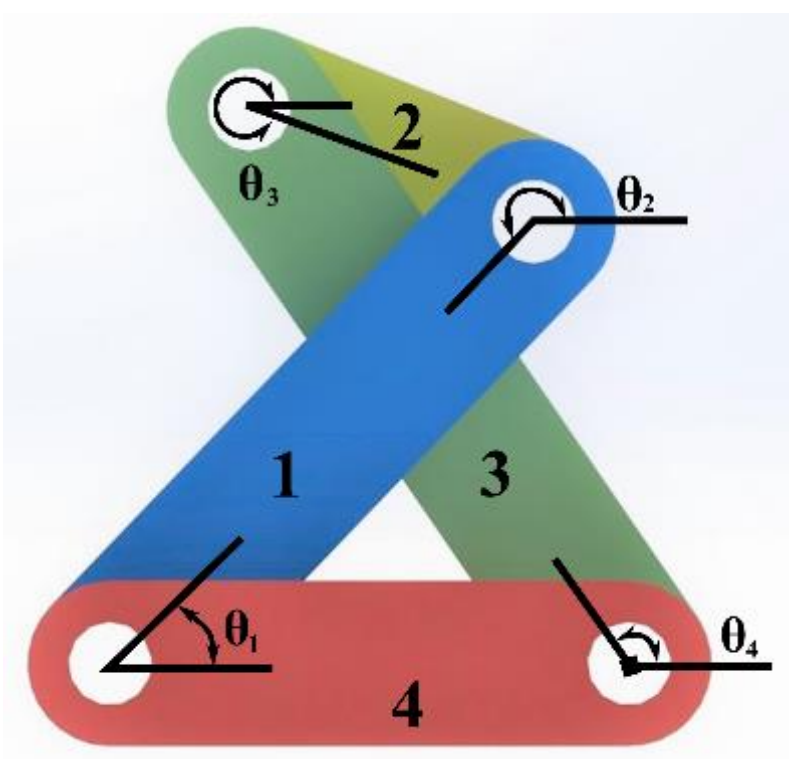

Figure 52 - Four-bar linkage used in the proposed design showing link designation, angle designation, and the coordinate system for the following equations.

For n number of moving bodies, there are $\mathrm{N}$ number of instantaneous centers of rotation such that

$$
N=\frac{n(n-1)}{2}
$$

From Equation 12, a four-bar linkage has six ICR, four of which are the joints where two links meet. This leaves two ICR that are not explicitly formed at joints; however, we are only interested in the ICR that is created by the crossing of the links. 
This ICR is of interest in particular because any force that creates rotation about the joint need to pass through this point.

This is the ICR formed by the intersection of the lines created from links 1 and 3, where link 1 is the PCL, link 3 represents the ACL, and links 2 and 4 represent the fibular end and tibial head respectively. This closed link configuration is defined as four vectors in a closed polygon where the sum is zero, written as

$$
\overrightarrow{A B}+\overrightarrow{B C}+\overrightarrow{C D}+\overrightarrow{D A}=0
$$

However, because $\overrightarrow{D A}$ is parallel to the origin it is reduced to just the length $\mathrm{L}_{4}$.

Then vector equation may be rewritten in complex-number form such that

$$
L_{1} e^{i \theta_{1}}+L_{2} e^{i \theta_{2}}+L_{3} e^{i \theta_{3}}+L_{4}
$$

Next Equation 14 is separated into the real and imaginary parts

$$
\begin{aligned}
& L_{1} \cos \left(\theta_{1}\right)=L_{3} \cos \left(\theta_{4}\right)+L_{2} \cos \left(\theta_{3}\right)+L_{4} \\
& L_{3} \sin \left(\theta_{1}\right)=L_{3} \sin \left(\theta_{4}\right)+L_{2} \sin \left(\theta_{3}\right)
\end{aligned}
$$

Solving to find the intersection of the two links, we obtain the set of equations for the instantaneous center of rotation given in $\mathrm{x}$ and $\mathrm{y}$ coordinates

$$
\begin{aligned}
& x=L_{4} \frac{\tan \left(\theta_{4}\right)}{\tan \left(\theta_{4}\right)-\tan \left(\theta_{1}\right)} \\
& y=L_{4} \frac{\tan \left(\theta_{4}\right) * \tan \left(\theta_{1}\right)}{\tan \left(\theta_{4}\right)-\tan \left(\theta_{1}\right)}
\end{aligned}
$$


The length of the links which correspond to our designed joint are [L1, L2, L3, $\mathrm{L} 4]=[1.85,0.92,2.03,1.57]$ given in inches. The ICR only moves \pm 0.2 inches out of plane from the desired line of action and has a maximum overall moment arm of 2.7 inches prior to the addition of a patella (Fig 53).

The designed joint is only 2.8 inches wide and has a range of motion of 151 degrees; this is roughly the same size and range of motion of the human knee. In a fully extended position, such as when standing, the joint will not rotate past that point so that no extra energy is needed to keep the joint in that position. This feature, in conjunction with the large moment arm, should greatly reduce the energy requirements of a robot when compared to a robot using a non-locking pin joint at the knee.

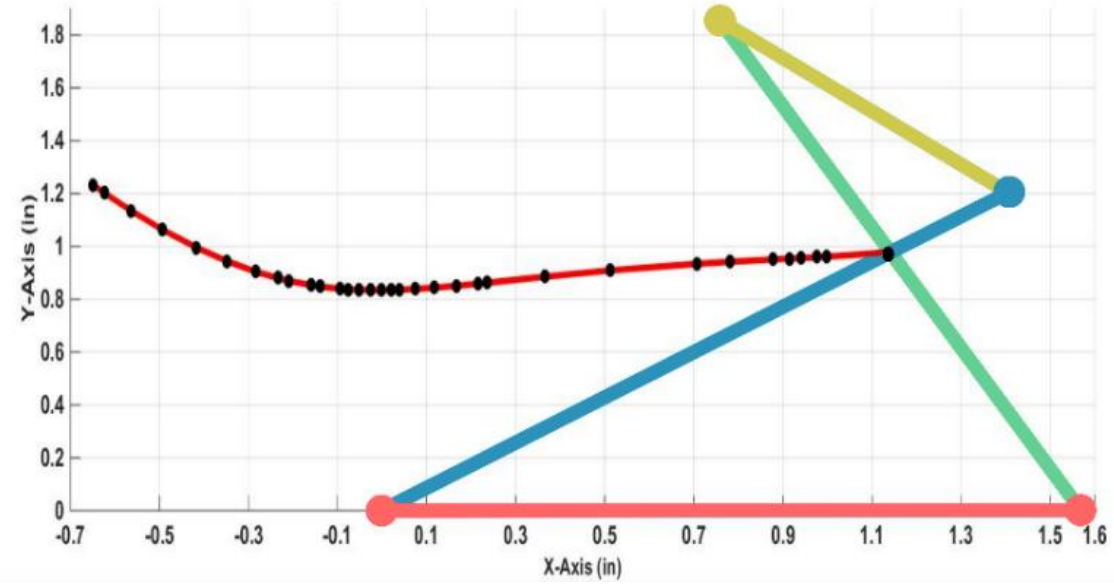

Figure 53 - Instantaneous center of rotation plot of proposed joint for the full range of motion.

I created a motion study to determine the sliding to rolling ratio of the joint using SolidWorks (Fig 54). First, the travel arc length of the tibia $\left(\Delta \mathrm{L}_{\mathrm{T}}\right)$ and the travel arc length of the femur $\left(\Delta \mathrm{L}_{\mathrm{F}}\right)$ are defined as 


$$
\begin{array}{r}
\Delta L_{T}(\theta)=C_{T f}(\theta)-C_{T i}(\theta) \\
\Delta L_{F}(\theta)=C_{F f}(\theta)-C_{F i}(\theta)
\end{array}
$$

where $\mathrm{C}_{\mathrm{Ti}}$ and $\mathrm{C}_{\mathrm{Fi}}$ are the initial contact points and $\mathrm{C}_{\mathrm{Tf}}$ and $\mathrm{C}_{\mathrm{Ff}}$ are the final contact points of the tibia and femur given an incremental flexion angle $(\theta)$.

Once the travel arc length is calculated, the sliding to rolling ratio (SR) is calculated by

$$
S R(\theta)=\frac{\Delta L_{T}(\theta)-\Delta L_{F}(\theta)}{\Delta L_{T}(\theta)}
$$

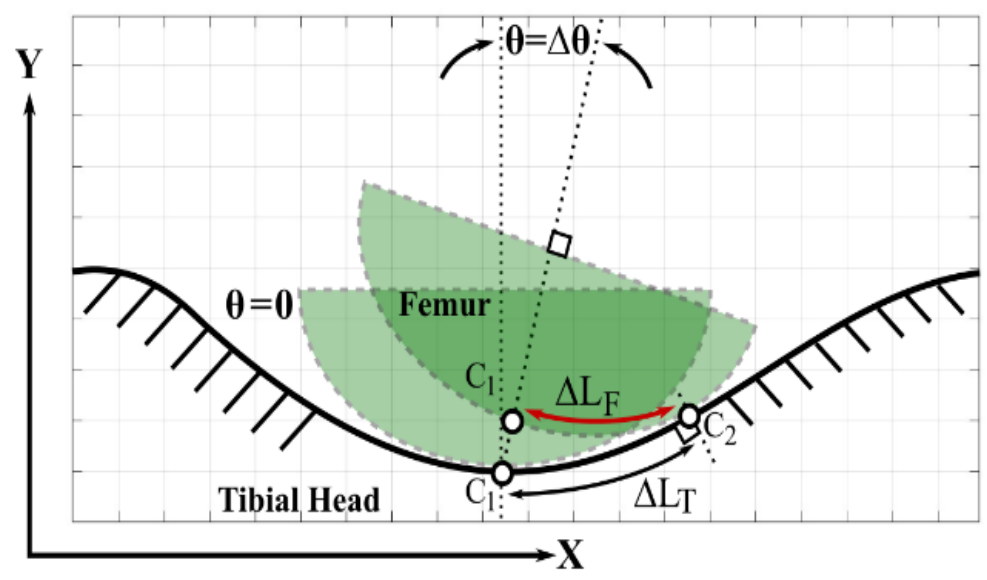

Figure 54 - Kinematics of sliding to rolling ratio from initial contact point $\mathrm{Cl}$ to $\mathrm{C} 2$

A SolidWorks Motion Analysis simulation was done to determine the sliding to rolling ratio with regard to flexion and was calculated at 0.5 -degree intervals through the full range of motion of the joint (Fig. 55). The ratio of sliding to rolling of the proposed joint, on average is 0.079 with a minimum value of 0.018 and a maximum value of 0.28 . 


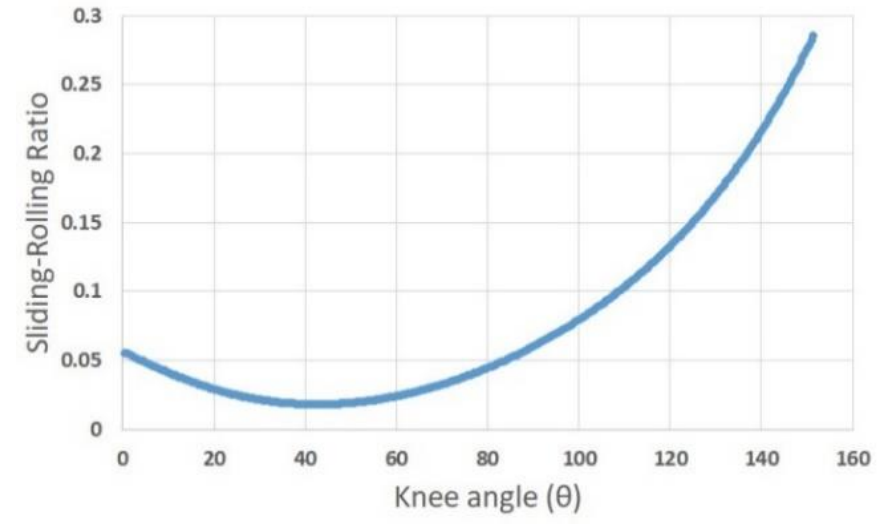

Figure 55 - Sliding to rolling ratio over the designed joints range of motion where zero is a pure rolling condition and one is a pure sliding condition, which increases wear.

\section{3 - Ankle and foot}

This section will focus primarily on the construction instead of the theoretical behavior of the joint because the hip and ankle joint share the same design.

\subsection{1 - Version 1}

The ankle is approximated as a ball and socket joint; the talus has a ball along with the connections needed to anchor the pneumatic actuator to the joint (Fig 56). 


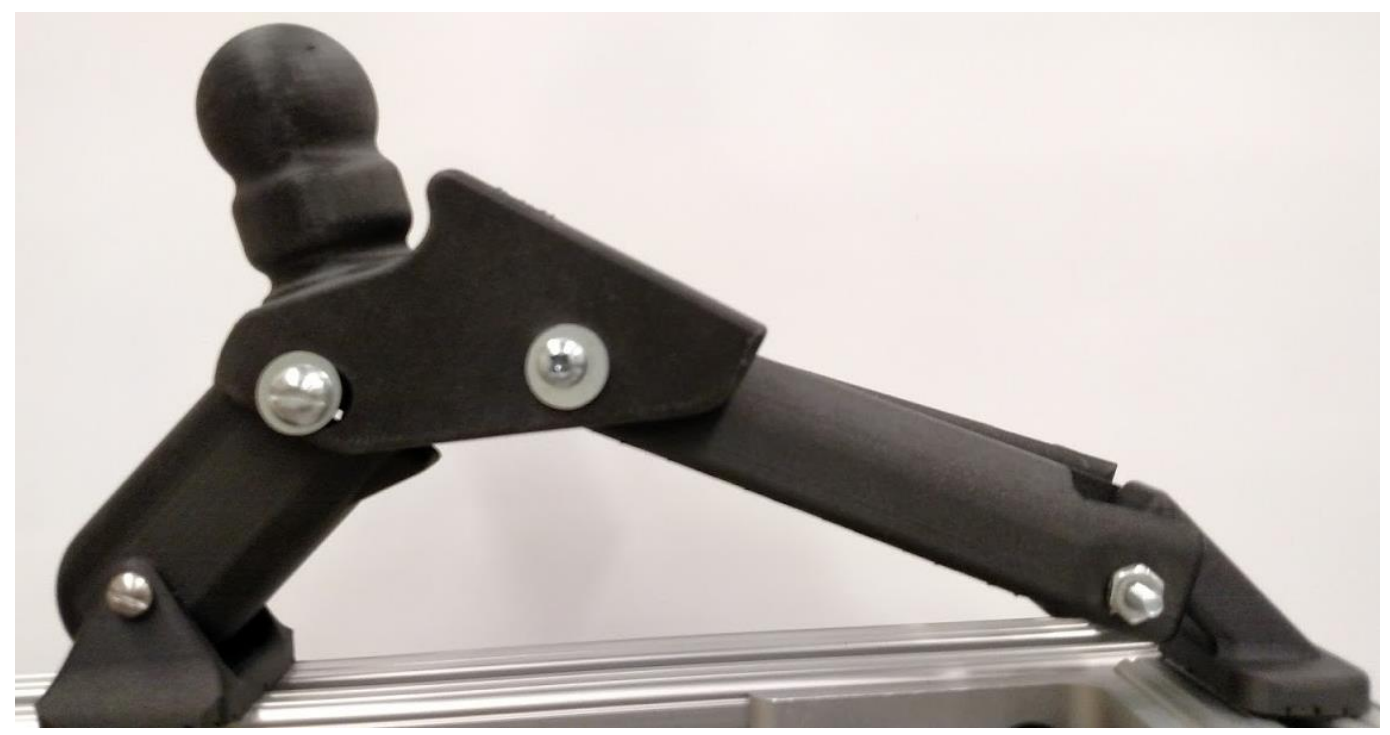

Figure $56-3 D$ printed version 1 of the foot as seen laterally

A lever arm that was added to the calcaneus to mimic the lever arm the calcaneus has in the foot (Fig. 57). This addition better mimics the human foot because the calcaneus applies a force in the same manner; however, the design leaves the pneumatic cylinder that would act as a spring element unprotected. This pneumatic cylinder would be located just below the lever arm; the attachment point was not included, because this was just a test print.



Figure 57 - Distal view of version 1 of the 3D printed foot showing the lever arm addition on the calcaneus 
There only needed to be two toes instead of the five we have because the foot behaves as two separate sections [29]. This meant that the design could be simplified, which also means that it could meet the requirement for it being under-actuated and is shown in the anterior view below in Fig. 58.

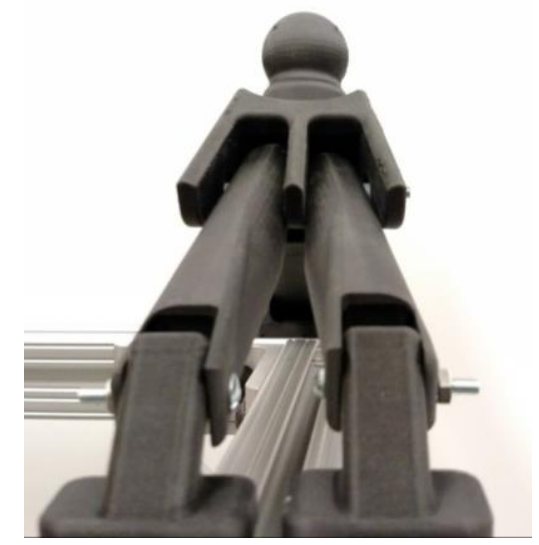

Figure 58 - Anterior view of the $3 D$ printed version 1 foot showing the two toes

\subsection{2 - Version 2}

The second foot design created a more natural foot while also creating a larger footprint (Fig. 59). Furthermore, while almost the entire foot is rounded, drop-testing shows that the design is very resilient to tipping and is much more stable than the previous design. 


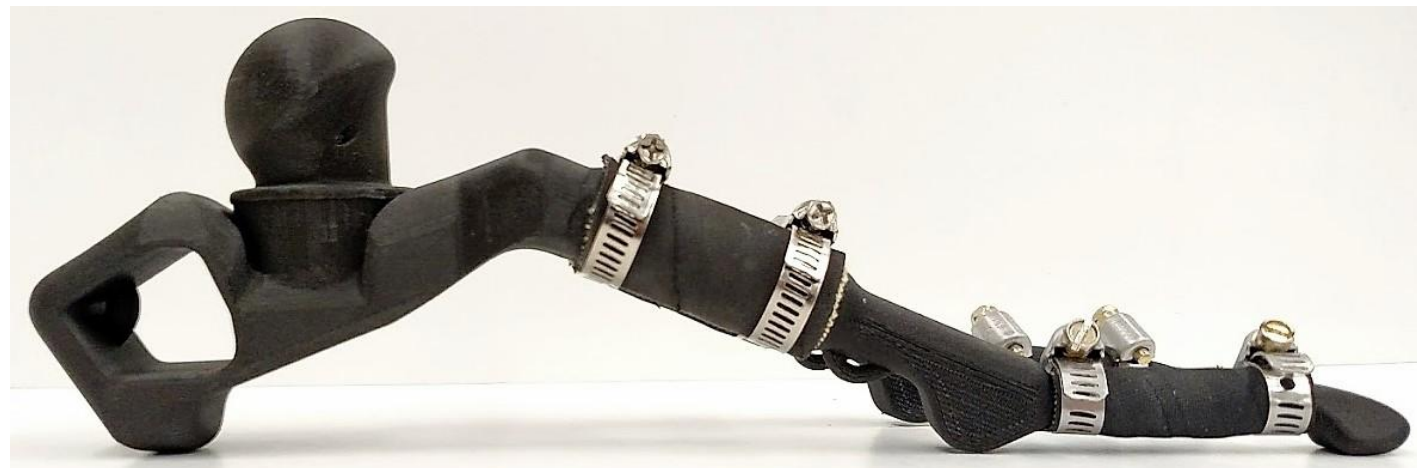

Figure 59 - Foot design version 2 seen laterally after the assembly using the pneumatic actuators as synovial joints

This design also included two hollow tubes inside the printed foot bones that allowed air to inflate all of the joints from a single connection at the ankle (Fig. 60).

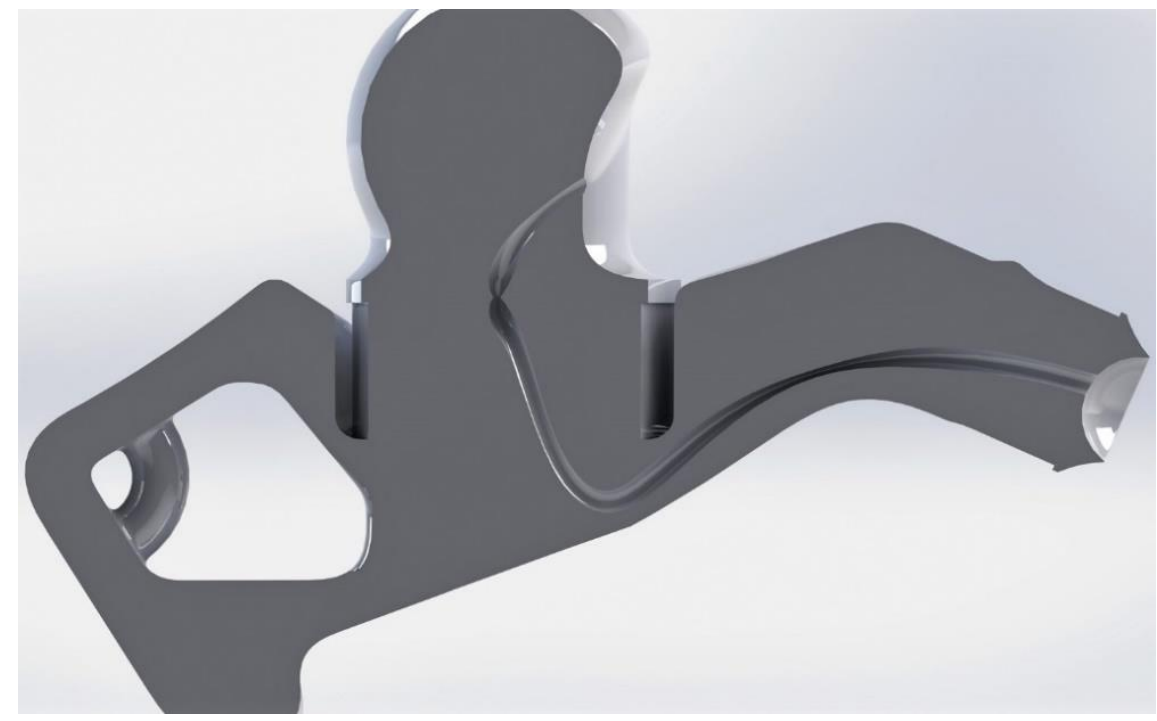

Figure 60 - Section view of the talus in SolidWorks showing the tube inside the model that lets air flow down into the other joints of the foot 
Next, the ankle joint needed to be tested to insure that the gap created was large enough to accommodate the pneumatic actuator. Figure 61 shows the full ankle and foot joint assembled with the pneumatic actuators.

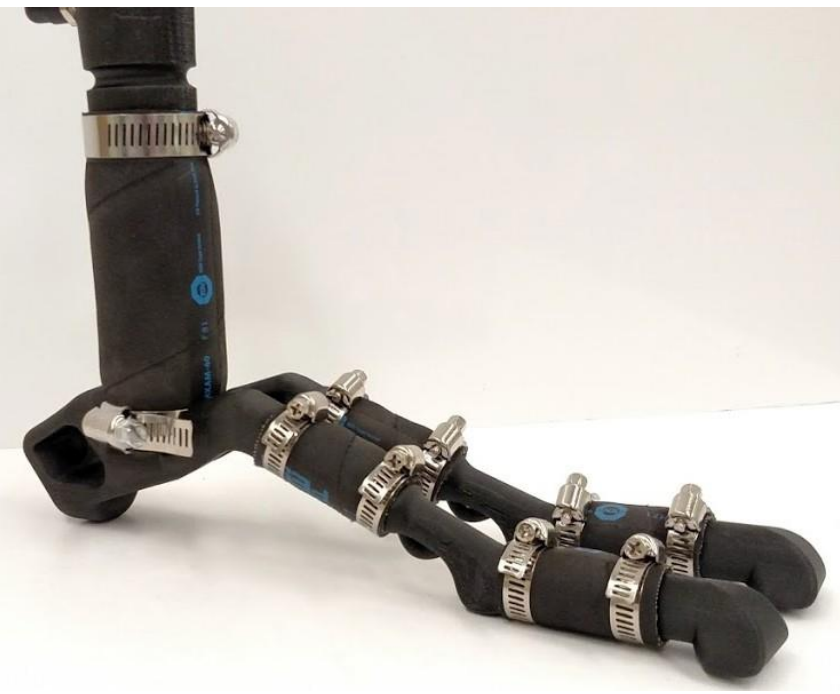

Figure $61-3 D$ Printed foot version 2 with the pneumatic actuator sleeve for the ankle added 


\section{Chapter 6}

\section{Discussion}

With respect to the hip and ankle joints, both joint stiffness and damping is expected to change as the angle of the joint changes, due to the increase in the curvature of the pneumatic actuator and the associated change in pressure, this change mimics the observed behavior of the human ankle [48-51]. This behavior should help stabilize a bipedal robot just as it helps stabilize humans.

Additionally, the joints should exhibit increased stability as the angle of the joint increases because the stiffness of the joint is expected to increase as a result of bending. This means the joint is expected to behave closer to a rigid joint as the angle increases; this too should be beneficial in robotic control.

Because braided pneumatic actuators have a protective fiber braid around the air bladder, should the air bladder puncture or become damaged, if the joint is in tension, the fiber braid should support the load which should help limit damage to the system as a whole. Conversely, if the air bladder fails and the joints are in tension, the fiber wrapping should mechanically limit the joint movement and while the system dynamics of the joint have changed, as long as the load applied to the fiber braid does not exceed its tensile strength the joint should remain semi-functional.

With respect to the knee, the comparison of the ICR curve with one developed by Fekete et al. [50] demonstrate that the placement of the curve is similar to the placement of the ICR curve of the human knee. Due to the compliance of tendons in the human 
knee, unlike the solid links used in the current design, the ICR curve of a human knee is more convex than the ICR curve of the designed knee. However, the placement of the curve is similar to the placement of the ICR curve of the human knee and the flattened ICR curve of the designed joint has desirable properties with respect to the force requirements to create motion.

There are too many variables to determine the sliding to rolling ratio of the human knee in vivo so we cannot confidently include a graphical comparison. However, current research on the subject suggests the sliding to rolling ratio of the human knee is between $0.3-0.46[46,52]$.

By mimicking the design of the human knee, the designed joint is comparable in size while having several of the knees' desirable properties. This is in part due to the complex geometry that was obtained from 3D printing instead of using traditional methods. 3D printing also reduced the time of construction to just over one day as compared to potentially weeks of traditional machining.

Because the joint is made primarily from a nylon-carbon fiber chop and has a continuous carbon fiber inlay, the joint has exceptional strength giving it functionally, but weighs just 0.66 pounds. Furthermore, the majority of the weight of the joint is due to the steel fastening hardware added internally to the design mid-print and not from the print material itself.

One of the goals in the design of our knee joint was to reduce wear, with an average sliding to rolling ratio of 0.079 ; the joint has a significantly lower sliding to 
rolling ratio than the human knee. By having continuous contact of the loading faces, the load applied to the pins is minimized and the addition of the locking extension on the rear of the tibia transfers a majority of the forces that would be placed on the pins at full flexion. By removing most of the loading forces from the pin connections, the joint will be less likely to suffer from a catastrophic failure and should last significantly longer than the traditional pin joint.

Reynolds et al. [53] using a sudden reduction in load with constant pressure experimentally determined the independent estimates for the spring coefficient $(\mathrm{K})$ and the damping coefficient (B) of braided pneumatic actuators. They determined that both the increase linearly with the change in pressure and that B and $\mathrm{K}$ could be functions of loading. Furthermore, that as the applied load increases damping of the system increases and the system becomes overdamped, however when the load decreases the system acts closer to a critically damped system.

This behavior should help increase the controllability of the system because the system will not oscillate due to perturbations. This also means that damping is expected to increase as the joint angle increases, which should further help stabilize the system. The proposed ankle joint should be adjusted to match closely with the human range of motion of the ankle as shown in table 4.

Table 4 - Average ankle joint range of motion

\begin{tabular}{l|ll}
\multicolumn{2}{l}{ Motion } & Range (degrees) \\
\hline Ankle joint & & \\
& Planter Flexion/ Dorsiflexion & $59-71$ \\
& Inversion/Eversion & $42-56$
\end{tabular}


Future work concerning the hip and ankle joints should include experimentally testing the relationship between the length of the pneumatic actuator, the joint gap after pressurization, initial pressure of the actuator, and the remaining volume inside the actuator to create a more complete model of the joint.

Once a more complete model is created, testing should be done using alternative pneumatic actuators constructed from materials with increased elasticity and flexibility. These properties should be adjusted using this new model in conjunction with the mechanical and material properties of the joints they are mimicking.

Future work for the knee should involve wear testing and further material property testing to verify the material properties data given from MarkForged. This will help better characterize the joint lifetime and failure modes. 


\section{Bibliography}

1. Saad Mukras, Nam H. Kim, Nathan A. Mauntler, Tony L. Schmitz, W. Gregory Sawyer: Analysis of planar multibody systems with revolute joint wear. Wear. 268, 643-652 (2010).

2. Asano, Y., Mizoguchi, H., Kozuki, T., Motegi, Y., Urata, J., Nakanishi, Y., Okada, K., Inaba, M.: Achievement of Twist Squat by Musculoskeletal Humanoid with Screw-Home Mechanism. IEEE. (2013).

3. Appolinaire C. Etoundi: A bio-inspired condylar hinge joint for mobile robots. International Conference on Intelligent Robots and Systems. (2011).

4. Burgess, S., Etoundi, A.: Performance Maps for a Bio-Inspired Robotic Condylar Hinge Joint. Journal of Mechanical Design. 136, 7 (2014).

5. Khan, H., Featherstone, R., Caldwell, D.G., Semini, C.: Bio-inspired knee joint mechanism for a hydraulic quadruped robot. In: 2015 6th International Conference on Automation, Robotics and Applications (ICARA). pp. 325-331 (2015).

6. Hobon, M., Elyaaqoubi, N.L., Abba, G.: Quasi optimal sagittal gait of a biped robot with a new structure of knee joint. Robotics and Autonomous Systems. 62, 436-445 (2014).

7. Susan Standring: Gray's Anatomy. Elsevier (2015).

8. Pamela K. Levangie, Cynthia C. Norkin: Joint Structure and Function: A Comprehensive Analysis Fifth Edition. F. A. Davis Company (2011).

9. Hewitt, J., Guilak, F., Glisson, R., Vail, T.P.: Regional material properties of the human hip joint capsule ligaments. Journal of Orthopaedic Research. 19, 359-364 (2001).

10. van Arkel, R.J., Amis, A.A., Cobb, J.P., Jeffers, J.R.T.: The capsular ligaments provide more hip rotational restraint than the acetabular labrum and the ligamentum teres: an experimental study. The Bone \& Joint Journal. 97-B, 484-491 (2015).

11. Joseph M. Mansour: Biomechanics of Cartilage. In: Biomechanical Principles.

12. Halonen, K.S., Mononen, M.E., Jurvelin, J.S., T€oyr€as, J., Kłodowski, A., Kulmala, J.-P., Korhonen, R.K.: Importance of Patella, Quadriceps Forces, and Depthwise Cartilage Structure on Knee Joint Motion and Cartilage Response During Gait. Journal of Biomechanical Engineering. 138, (2016).

13. Michael Harty: The Anatomy of the Hip Joint. Surgery of the Hip Joint. 45-74 (1984).

14. Hodge, W.A., Fijan, R.S., Carlson, K.L., Burgess, R.G., Harris, W.H., Mann, R.W.: Contact pressures in the human hip joint measured in vivo. Proceedings of the National Academy of Sciences. 83, 2879-2883 (1986).

15. Stewart C. Wang, Chris Brede, David Lange, Craig S. Poster, Aaron W. Lange, Carla Kohoyda-Inglis, Mark R. Sochor, Kyros Ipaktchi, Stephen A. Rowe: Gender Differences in Hip Anatomy: Possible Implications for Injury Tolerance in Frontal Collisions. Association for the Advancement of Automotive Medicine. 287-301 (2004). 
16. American College of Sports Medicine, http://www.acsm.org/.

17. Horton, M.G., Hall, T.L.: Quadriceps Femoris Muscle Angle: Normal Values and Relationships with Gender and Selected Skeletal Measures. Phys Ther. 69, 897-901 (1989).

18. Mohammad-Jafar Emami, Mohammad-Hossein Ghahramani, Farzad Abdinejad, Hamid Namazi: Q-angle: An Invaluable Parameter for Evaluation of Anterior Knee Pain. Archives of Iranian Medicine. 10, (2007).

19. Hugo Machado Sanchez, Eliane Gouveia de Morais Sanchez, Mario Antonio Baraúna, Roberto Sérgio de Tavares Cant: Evaluation of Q angle in differents static postures. Acta Ortop Bras. 22, 325-329 (2014).

20. Jawad F. Abulhasan, Michael J. Grey: Anatomy and Physiology of Knee Stability. Journal of Functional Morphology and Kinesiology. 2, (2017).

21. S. A. Shahane, C. Ibbotson, R. Strachan, D. R. Bickerstaff: The popliteofibular ligament - An anatomical study of the posterolateral corner of the knee. The Journal of Bone and Joint Surgery. (1998).

22. Erin M. Parsons, Albert O. Gee, Charles Spiekerman, Peter R. Cavanagh: The Biomechanical Function of the Anterolateral Ligament of the Knee. The American Journal of Sports Medicine. 43, 669-674 (2015).

23. Yuki Asano, Hironori Mizoguchi, Masahiko Osada, Toyotaka Kozuki, Junichi Urata, Tamon Izawa, Yuto Nakanishi, Kei Okada, Masayuki Inaba: Biomimetic Design of Musculoskeletal Humanoid Knee Joint with Patella and Screw-Home Mechanism. Robotics and Biomimetics (ROBIO), 2011 IEEE International Conference on Robotics and Biomimetics. (2011).

24. Bhaskar Kumar, Srinivasa Rao, S. K. Sundara siva rao: Biomechanics of knee joint - A review. Frontiers of Mechanical Engineering. 10, 176-186 (2015).

25. Donatelli, R.A.: Normal biomechanics of the foot and ankle. J Orthop Sports Phys Ther. 7, 91-95 (1985).

26. Milner, C.E., Soames, R.W.: Anatomy of the Collateral Ligaments of the Human Ankle Joint. Foot \& Ankle International. 19, 757-760 (1998).

27. Kelikian, A.S., Sarrafian, S.K.: Sarrafian's Anatomy of the Foot and Ankle: Descriptive, Topographic, Functional. Lippincott Williams \& Wilkins (2011).

28. Chitsazan, A., Rouhi, G., Abbasi, M., Pezeshki, S., Tavakoli, S.A.H.: Assessment of stress distribution in ankle joint: simultaneous application of experimental and finite element methods. International Journal of Experimental and Computational Biomechanics. 3, 45 (2015).

29. Golanó, P., Vega, J., de Leeuw, P.A.J., Malagelada, F., Manzanares, M.C., Götzens, V., van Dijk, C.N.: Anatomy of the ankle ligaments: a pictorial essay. Knee Surgery, Sports Traumatology, Arthroscopy. 24, 944-956 (2016).

30. Scott, S.H., Winter, D.A.: Biomechanical model of the human foot: Kinematics and kinetics during the stance phase of walking. Journal of Biomechanics. 26, 1091-1104 (1993).

31. Mark Two - Markforged, https://markforged.com/mark-two/.

32. Carbon Fiber, http://support.markforged.com/hc/en-us/articles/211439563-CarbonFiber. 
33. Kevlar, http://support.markforged.com/hc/en-us/articles/209934366-Kevlar.

34. Standard Fiberglass, http://support.markforged.com/hc/en-us/articles/209934386Standard-Fiberglass.

35. High-Strength, High-Temperature Fiberglass, http://support.markforged.com/hc/enus/articles/211358483-High-Strength-High-Temperature-Fiberglass.

36. Bücking, T.M., Hill, E.R., Robertson, J.L., Maneas, E., Plumb, A.A., Nikitichev, D.I.: From medical imaging data to 3D printed anatomical models. PLOS ONE. 12, e0178540 (2017).

37. Pelvis and Hip - CT scan, https://www.embodi3d.com/files/file/11745-pelvis-andhip-ct-scan/.

38. Kazemi, S.M., Qoreishy, M., Keipourfard, A., Sajjadi, M.M., Shokraneh, S.: Effects of Hip Geometry on Fracture Patterns of Proximal Femur. Arch Bone Jt Surg. 4, 248-252 (2016).

39. Hui-Hui Wu, null, Dong Wang, null, An-Bang Ma, null, Dong-Yun Gu, null: Hip joint geometry effects on cartilage contact stresses during a gait cycle. Conf Proc IEEE Eng Med Biol Soc. 2016, 6038-6041 (2016).

40. Hartel, M.J., Petersik, A., Schmidt, A., Kendoff, D., Nüchtern, J., Rueger, J.M., Lehmann, W., Grossterlinden, L.G.: Determination of Femoral Neck Angle and Torsion Angle Utilizing a Novel Three-Dimensional Modeling and Analytical Technology Based on CT Datasets. PLoS One. 11, (2016).

41. Colbrunn, R.W., Nelson, G.M., Quinn, R.D.: Modeling of braided pneumatic actuators for robotic control. In: Proceedings 2001 IEEE/RSJ International Conference on Intelligent Robots and Systems. Expanding the Societal Role of Robotics in the the Next Millennium (Cat. No.01CH37180). pp. 1964-1970 vol.4 (2001).

42. Chou, C.-P., Hannaford, B.: Measurement and modeling of McKibben pneumatic artificial muscles. IEEE Transactions on Robotics and Automation. 12, 90-102 (1996).

43. J. M. Soucie, C. Wang, A. Forsyth, S. Funk, M. Denny, K. E. Roach, D. Boone: Range of motion measurements: reference values and a database for comparison studies. Haemophilia. 500-507 (2011).

44. Roaas, A., Andersson, G.B.J.: Normal Range of Motion of the Hip, Knee and Ankle Joints in Male Subjects, 30-40 Years of Age. Acta Orthopaedica Scandinavica. 53, 205-208 (1982).

45. Kouyoumdjian, P., Coulomb, R., Sanchez, T., Asencio, G.: Clinical evaluation of hip joint rotation range of motion in adults. Orthopaedics \& Traumatology: Surgery \& Research. 98, 17-23 (2012).

46. Fekete, G., De Baets, P., Abdel Wahab, M., Csizmadia, B., Katona, G., VanegasUseche, L., Solanilla, J.: Sliding-Rolling Ratio during Deep Squat with Regard to Different Knee Prostheses. Acta Polytechnica Hungarica. 9, 20 (2012).

47. Fekete, G.: Kinetics and Kinematics of the Human Knee Joint under Standard and Non-Standard Squat Movement, (2013).

48. Amiri, P., Kearney, R.E.: Ankle intrinsic stiffness is modulated by postural sway. Presented at the July (2017). 
49. Berno J. E. Misgeld, Tony Zhang, Markus J. Lüken, Steffen Leonhardt: Model-Based Estimation of Ankle Joint Stiffness. (2017).

50. Guarin, D.L., Kearney, R.E.: Time-varying identification of ankle dynamic joint stiffness during movement with constant muscle activation. Presented at the August (2015).

51. Hettich, G., Assländer, L., Gollhofer, A., Mergner, T.: Human hip-ankle coordination emerging from multisensory feedback control. Human Movement Science. 37, 123-146 (2014).

52. Masouros, S.D., Bull, A.M.J., Amis, A.A.: Biomechanics of the knee joint. ORTHOPAEDICS AND TRAUMA. 24, 84-91 (2010).

53. Reynolds, D.B., Repperger, D.W., Phillips, C.A., Bandry, G.: Modeling the Dynamic Characteristics of Pneumatic Muscle. Annals of Biomedical Engineering. 31, 310317 (2003). 